\title{
Three-gluon vertex in arbitrary gauge and dimension
}

\author{
A. I. Davydychev* and P. Osland \\ Department of Physics, University of Bergen, Allégaten 55, N-5007 Bergen, Norway \\ O. V. Tarasov \\ Fakultät für Physik, Universität Bielefeld, D-33615, Bielefeld 1, Germany \\ and NORDITA, Blegdamsvej 17, DK-2100 Copenhagen Ф, Denmark \\ (Received 20 May 1996)
}

\begin{abstract}
One-loop off-shell contributions to the three-gluon vertex are calculated, in an arbitrary covariant gauge and in arbitrary space-time dimensions, including quark-loop contributions (with massless quarks). It is shown how one can get the results for all on-shell limits of interest directly from the general off-shell expression. The corresponding general expressions for the one-loop ghost-gluon vertex are also obtained. They allow for a check of consistency with the Ward-Slavnov-Taylor identity. [S0556-2821(96)05818-3]

PACS number(s): 11.15.Bt, 11.10.Kk, 12.38.Bx
\end{abstract}

\section{INTRODUCTION}

The three-gluon coupling is perhaps the most obvious manifestation of the non-Abelian aspect of quantum chromodynamics [1] (see also the reviews [2,3]). Implicitly, it has been studied experimentally through the observed running of the coupling constant [4]. The associated Casimir invariant has even been measured directly in studies of four-jet events at the CERN $e^{+} e^{-}$collider LEP [5], the SU(3) group being consistent with the data.

Apart from being a standard object of consideration in textbooks on quantum field theory and QCD (see, e.g., [6-8]), the perturbative corrections to gluonic vertices are also very important in real physical calculations, such as multijet production at the hadron colliders (see, e.g., [9,3] and references therein). At the present level of accuracy, one needs to perform not only calculations with on-shell external particles; there are also contributions where general off-shell results are needed.

One of the original reasons the three-gluon vertex was studied was the belief that its infrared properties might shed light on the mechanism of confinement. In these studies, different approaches were used, some of which are discussed in the review [10] (and references therein).

For special cases, the one-loop results for the three-gluon coupling have been known for many years. Celmaster and Gonsalves (CG) presented in 1979 [11] the one-loop result for the vertex, for off-shell gluons, restricted to the symmetric case, $p_{1}^{2}=p_{2}^{2}=p_{3}^{3}$, in an arbitrary covariant gauge. ${ }^{1}$ Ball and Chiu (BC) then in 1980 considered the general off-shell

\footnotetext{
*Permanent address: Institute for Nuclear Physics, Moscow State University, 119899, Moscow, Russia. Electronic address: davyd@vsfys1.fi.uib.no

Electronic address: Per.Osland@fi.uib.no

\#n leave from Joint Institute for Nuclear Research, 141980, Dubna, Russia. Present address: IfH, DESY-Zeuthen, Platanenallee 6, D-15738 Zeuthen, Germany. Electronic address: tarasov@ifh.de ${ }^{1}$ The result of [11] was also confirmed by Pascual and Tarrach [12].
}

case, but restricted to the Feynman gauge [13]. Later, various on-shell results were also given, by Brandt and Frenkel (BF) [14], restricted to the infrared-singular parts only (in an arbitrary covariant gauge), and by Nowak, Praszałowicz, and Słomiński (NPS) [15], who also gave the finite parts for the case of two gluons being on shell (in the Feynman gauge). An overview of these results is given in Table I.

From Table I one can see that, even if we consider the results in (or around) four dimensions, there are still several " white spots." They correspond not only to the most general case (the lower left corner), but also to some other cases when the results are missing, either for quark loop contributions or for the finite parts. The aim of the present paper is to cover all such remaining spots (for the case when massless quarks are considered). Moreover, we present results which are valid for an arbitrary value of the space-time dimension. Apart from the three-gluon vertex itself, we also consider the ghost-gluon vertex and two-point functions, to be able to check that all these expressions obey the Ward-SlavnovTaylor identity for the three-gluon vertex.

At the one-loop level, the simple and well-known Lorentz structure of the lowest-order coupling gets modified. In the general case, six tensor structures (and their permutations) are needed to decompose the three-gluon vertex [13]. Thus, six scalar functions multiplying these tensor structures are to be calculated. These scalar functions depend on the gauge parameter, the space-time dimension, and the kinematical invariants $\left(p_{1}^{2}, p_{2}^{2}, p_{3}^{2}\right)$.

There are several reasons why the one-loop results calculated in arbitrary gauge and dimension $n$ are of special interest: (i) Knowing the results in arbitrary gauge, one can explicitly keep track of gauge invariance for physical quantities; (ii) if one is interested in the two-loop calculation of the three-gluon coupling, one should know one-loop contributions in more detail; (iii) results in arbitrary dimension make it possible to consider all on-shell limits (when some $p_{i}^{2}=0$ ) directly from these expressions (see Sec. IV), and this is impossible if one only has the results valid around four dimensions; (iv) QCD is also a theory of interest in three and two dimensions (see, e.g., [16] and the review $[17])$; and (v) as we shall see, the results for arbitrary dimen- 

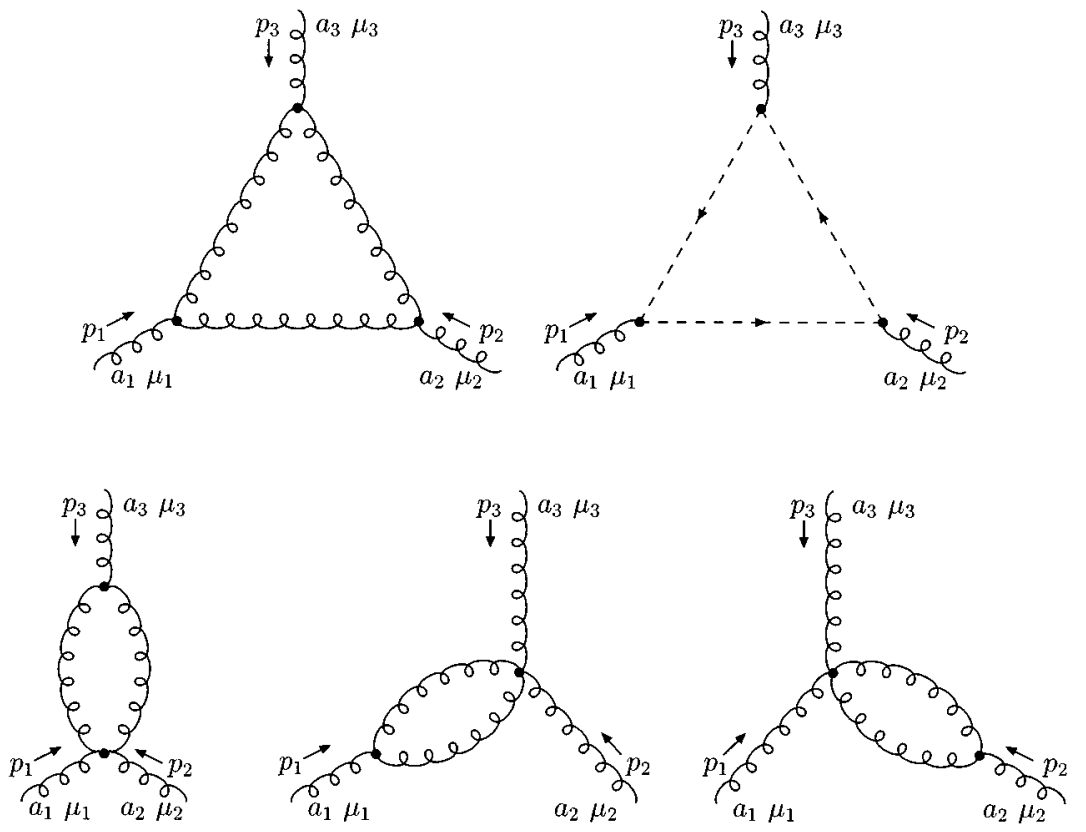

FIG. 1. One-loop three-gluon vertex diagrams.

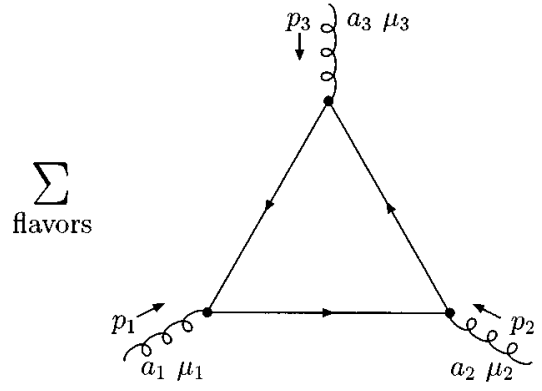

sion are not much more cumbersome than those considered around four dimensions (in some respects, they are even more transparent and instructive).

We note that in several papers the one-loop three-gluon vertex in axial-type gauges (including the light-cone gauge) was considered [18] (mainly divergent parts and special limits have been studied). The three-gluon vertex in the background field formalism was considered in Ref. [19], while the gauge-invariant vertex was studied in Ref. [20]. Moreover, there were some lattice calculations of the three-gluon vertex; see, e.g., Ref. [21]. We shall not address these issues here, but concentrate instead on the standard vertex in an arbitrary covariant gauge.

The paper is organized as follows. In Sec. II, we introduce the notation for the two- and three-point functions to be considered, and discuss their decomposition in terms of scalar functions as well as the corresponding Ward-Slavnov-Taylor identity. In Sec. III, we present the most general off-shell results for the three-gluon vertex. Section IV contains the corresponding expressions for all on-shell limits of interest. In Sec. V, we conclude with a summary and a discussion of the results. Then, we have several appendixes where some further results and technical details are presented, such as the formulas used to decompose the three-gluon vertex (Appen$\operatorname{dix} \mathrm{A}$ ), relevant results for the scalar integrals involved (Appendix B), results for the self-energies (Appendix C) and the ghost-gluon vertex (Appendix D), expressions for the on- shell limit $p_{3}^{2}=0$ in an arbitrary gauge (Appendix E), and also some results for $p_{1}^{2}=p_{2}^{2}=0$ (Appendix F).

\section{PRELIMINARIES}

The Yang-Mills term of the QCD Lagrangian yields the following well-known expression for the lowest-order threegluon vertex:

$$
\begin{aligned}
& -i g f^{a_{1} a_{2} a_{3}}\left[g_{\mu_{1} \mu_{2}}\left(p_{1}-p_{2}\right)_{\mu_{3}}+g_{\mu_{2} \mu_{3}}\left(p_{2}-p_{3}\right)_{\mu_{1}}\right. \\
& \left.\quad+g_{\mu_{3} \mu_{1}}\left(p_{3}-p_{1}\right)_{\mu_{2}}\right]
\end{aligned}
$$

where $p_{1}, p_{2}$, and $p_{3}$ are the momenta of the gluons, all of which are ingoing, $p_{1}+p_{2}+p_{3}=0$. In Eq. (2.1), the $f^{a_{1} a_{2} a_{3}}$ are the totally antisymmetric color structures corresponding to the adjoint representation of the gauge group. ${ }^{2}$ They can be extracted from the general three-gluon vertex by defining ${ }^{3}$

\footnotetext{
${ }^{2}$ Although the standard QCD Lagrangian corresponds to the $\mathrm{SU}(3)$ group, our results are valid for an arbitrary semisimple gauge group.

${ }^{3}$ In fact, also completely symmetric color structures $d^{a_{1} a_{2} a_{3}}$ might be considered, but they do not appear in the perturbative calculation of QCD three-point vertices at the one-loop level.
} 
TABLE I. Kinematics and gauges considered in other studies.

All momenta off-shell

Some momenta on-shell

\begin{tabular}{|c|c|c|c|c|}
\hline & General case & $p_{1}^{2}=p_{2}^{2}=p_{3}^{2}$ & $p_{3}^{2}=0$ & $p_{1}^{2}=p_{2}^{2}=0$ \\
\hline $\begin{array}{l}\text { Feynman } \\
\text { gauge }\end{array}$ & $\begin{array}{l}\text { BC [13], } \\
\text { Eq. (3.3) } \\
\text { no quarks }\end{array}$ & $\begin{array}{l}\text { special case } \\
\text { of } \mathrm{CG}[11]\end{array}$ & $\begin{array}{c}\text { special case } \\
\text { of } \mathrm{BF}[14]\end{array}$ & $\begin{array}{l}\text { NPS [15], } \\
\text { Appendix B }\end{array}$ \\
\hline $\begin{array}{l}\text { Arbitrary } \\
\text { covariant } \\
\text { guage }\end{array}$ & & $\begin{array}{l}\text { CG [11], } \\
\text { Eq. (14) }\end{array}$ & $\begin{array}{c}\text { BF [14], } \\
\text { Eq. }(25), \\
\text { no quarks, } \\
\text { no finite parts }\end{array}$ & $\begin{array}{c}\text { BF [14], } \\
\text { Eq. (30), } \\
\text { no quarks, } \\
\text { no finite parts }\end{array}$ \\
\hline
\end{tabular}

$$
\Gamma_{\mu_{1} \mu_{2} \mu_{3}}^{a_{1} a_{2} a_{3}}\left(p_{1}, p_{2}, p_{3}\right) \equiv-i g f^{a_{1} a_{2} a_{3}} \Gamma_{\mu_{1} \mu_{2} \mu_{3}}\left(p_{1}, p_{2}, p_{3}\right) .
$$

Since the gluons are bosons, and since the color structures $f^{a_{1} a_{2} a_{3}}$ are antisymmetric, $\Gamma_{\mu_{1} \mu_{2} \mu_{3}}\left(p_{1}, p_{2}, p_{3}\right)$ must also be antisymmetric under any interchange of a pair of gluon momenta and the corresponding Lorentz indices.

The lowest-order gluon propagator is

$$
\delta^{a_{1} a_{2}} \frac{1}{p^{2}}\left(g_{\mu_{1} \mu_{2}}-\xi \frac{p_{\mu_{1}} p_{\mu_{2}}}{p^{2}}\right),
$$

where $\xi$ is the gauge parameter corresponding to a general covariant gauge, defined such that $\xi=0$ is the Feynman gauge. Here and henceforth, a causal prescription is understood, $1 / p^{2} \rightarrow 1 /\left(p^{2}+i 0\right)$.
When one calculates radiative corrections to the threegluon vertex (the corresponding one-loop diagrams are presented in Fig. 1), other tensor structures arise, in addition to the lowest-order expression (2.1), and the general tensor decomposition should be considered. If we take into account momentum conservation (only two of the external momenta are independent), 14 independent tensor structures carrying 3 Lorentz indices exist, and in general $\Gamma_{\mu_{1} \mu_{2} \mu_{3}}$ can be written as a sum of these tensors multiplied by scalar functions (see Appendix A). This decomposition is useful for extracting the corresponding scalar functions from the result of a calculation. Although bosonic symmetry of the vertex puts some conditions on the corresponding scalar functions, the explicit symmetry of the expression is broken, because one of the momenta was substituted in terms of two others.

To avoid this, one can use a more symmetric decomposition of the general three-gluon vertex, proposed by Ball and Chiu $^{4}[13]$ :

$$
\begin{aligned}
\Gamma_{\mu_{1} \mu_{2} \mu_{3}}\left(p_{1}, p_{2}, p_{3}\right)= & A\left(p_{1}^{2}, p_{2}^{2} ; p_{3}^{2}\right) g_{\mu_{1} \mu_{2}}\left(p_{1}-p_{2}\right)_{\mu_{3}}+B\left(p_{1}^{2}, p_{2}^{2} ; p_{3}^{2}\right) g_{\mu_{1} \mu_{2}}\left(p_{1}+p_{2}\right)_{\mu_{3}}-C\left(p_{1}^{2}, p_{2}^{2} ; p_{3}^{2}\right)\left[\left(p_{1} p_{2}\right) g_{\mu_{1} \mu_{2}}\right. \\
& \left.-p_{1 \mu_{2}} p_{2 \mu_{1}}\right]\left(p_{1}-p_{2}\right)_{\mu_{3}}+\frac{1}{3} S\left(p_{1}^{2}, p_{2}^{2}, p_{3}^{2}\right)\left(p_{1 \mu_{3}} p_{2 \mu_{1}} p_{3 \mu_{2}}+p_{1 \mu_{2}} p_{2 \mu_{3}} p_{3 \mu_{1}}\right)+F\left(p_{1}^{2}, p_{2}^{2} ; p_{3}^{2}\right) \\
& \times\left[\left(p_{1} p_{2}\right) g_{\mu_{1} \mu_{2}}-p_{1 \mu_{2}} p_{2 \mu_{1}}\right]\left[p_{1 \mu_{3}}\left(p_{2} p_{3}\right)-p_{2 \mu_{3}}\left(p_{1} p_{3}\right)\right]+H\left(p_{1}^{2}, p_{2}^{2}, p_{3}^{2}\right)\left\{-g_{\mu_{1} \mu_{2}}\left[p_{1 \mu_{3}}\left(p_{2} p_{3}\right)\right.\right. \\
& \left.\left.-p_{2 \mu_{3}}\left(p_{1} p_{3}\right)\right]+\frac{1}{3}\left(p_{1 \mu_{3}} p_{2 \mu_{1}} p_{3 \mu_{2}}-p_{1 \mu_{2}} p_{2 \mu_{3}} p_{3 \mu_{1}}\right)\right\} \\
& +\left\{\text { cyclic permutations of }\left(p_{1}, \mu_{1}\right),\left(p_{2}, \mu_{2}\right),\left(p_{3}, \mu_{3}\right)\right\} .
\end{aligned}
$$

Here, the $A, C$, and $F$ functions are symmetric in the first two arguments, the $H$ function is totally symmetric, and the $B$ function is antisymmetric in the first two arguments, while the $S$ function is antisymmetric with respect to interchange of any pair of arguments. Note that the contribution containing the $F$ and $H$ functions is totally transverse; i.e., it gives zero when contracted with any of $p_{1 \mu_{1}}, p_{2 \mu_{2}}$, or $p_{3 \mu_{3}}$.

Now, before proceeding further, we introduce some notation. For a quantity $X$ (e.g., any of the scalar functions contributing to the propagators or the vertices), we shall denote

\footnotetext{
${ }^{4}$ Another general decomposition of the three-gluon vertex was considered in Ref. [22].
}

the zero-loop-order contribution as $X^{(0)}$ and the one-looporder contribution as $X^{(1)}$. In this paper, as a rule,

$$
X^{(1)}=X^{(1, \xi)}+X^{(1, q)}
$$

where $X^{(1, \xi)}$ denotes a contribution of gluon and ghost loops in a general covariant gauge $(2.3)$ (in particular, $X^{(1,0)}$ corresponds to the Feynman gauge, $\xi=0)$, while $X^{(1, q)}$ represents the contribution of the quark loops.

For example, from Eq. (2.1) one can see that at the "zeroloop" level all the scalar functions involved in Eq. (2.4) vanish, except the $A$ function, which is

$$
A^{(0)}=1 \text {. }
$$




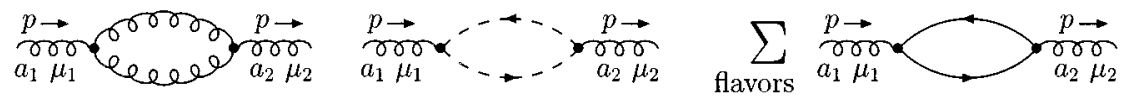

(a)

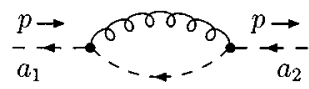

(b)
FIG. 2. (a) Gluon polarization operator diagrams and (b) ghost self-energy diagram.
In what follows, we shall also need to use some other QCD Green functions, including those involving the Faddeev-Popov ghosts. As in Eq. (2.4) we define the corresponding scalar structures following the notation of Ref. [13].

The gluon polarization operator is defined as

$$
\Pi_{\mu_{1} \mu_{2}}^{a_{1} a_{2}}(p) \equiv-\delta^{a_{1} a_{2}}\left(p^{2} g_{\mu_{1} \mu_{2}}-p_{\mu_{1}} p_{\mu_{2}}\right) J\left(p^{2}\right),
$$

while the ghost self-energy is

$$
\widetilde{\Pi}^{a_{1} a_{2}}\left(p^{2}\right)=\delta^{a_{1} a_{2}} p^{2} G\left(p^{2}\right) .
$$

The lowest-order results are $J^{(0)}=G^{(0)}=1$. The one-loop contributions to $\Pi_{\mu_{1} \mu_{2}}^{a_{1} a_{2}}(p)$ and $\widetilde{\Pi}^{a_{1} a_{2}}\left(p^{2}\right)$ are presented in Fig. 2 and can easily be calculated. The results (in arbitrary space-time dimension) can be found, e.g., in Ref. [8]. For completeness, we collect the relevant formulas in Appendix C.

The ghost-gluon vertex can be represented as

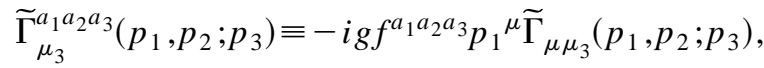

where $p_{1}$ is the out-ghost momentum, $p_{2}$ is the in-ghost momentum, and $p_{3}$ and $\mu_{3}$ are the momentum and the Lorentz index of the gluon (all momenta are ingoing). For $\widetilde{\Gamma}_{\mu \mu_{3}}$ we adopt the following decomposition, also used in [13]:

$$
\begin{aligned}
\widetilde{\Gamma}_{\mu \mu_{3}}\left(p_{1}, p_{2} ; p_{3}\right)= & g_{\mu \mu_{3}} a\left(p_{3}, p_{2}, p_{1}\right)-p_{3 \mu} p_{2 \mu_{3}} b\left(p_{3}, p_{2}, p_{1}\right) \\
& +p_{1 \mu} p_{3 \mu_{3}} c\left(p_{3}, p_{2}, p_{1}\right) \\
& +p_{3 \mu} p_{1 \mu_{3}} d\left(p_{3}, p_{2}, p_{1}\right) \\
& +p_{1 \mu} p_{1 \mu_{3}} e\left(p_{3}, p_{2}, p_{1}\right) .
\end{aligned}
$$

At the "zero-loop" level,

$$
\widetilde{\Gamma}_{\mu \mu_{3}}^{(0)}=g_{\mu \mu_{3}},
$$

and therefore all the scalar functions involved in Eq. (2.10) vanish at this order, except one, $a^{(0)}=1$. We shall also need the one-loop-order results for the ghost-gluon vertex in an arbitrary gauge (the corresponding diagrams are presented in Fig. 3). We have calculated one-loop contributions to all scalar functions occurring on the right-hand side (RHS) of Eq. (2.10); they are presented in Appendix D.

We need $\widetilde{\Gamma}_{\mu \mu_{3}}$ with two Lorentz indices, because this is what enters the Ward-Slavnov-Taylor identity for the threegluon vertex, which, in the covariant gauge, has the form (see, e.g., in $[2,13]$ )

$$
\begin{aligned}
& p_{3}^{\mu_{3}} \Gamma_{\mu_{1} \mu_{2} \mu_{3}}\left(p_{1}, p_{2}, p_{3}\right)=-J\left(p_{1}^{2}\right) G\left(p_{3}^{2}\right)\left(g_{\mu_{1}}{ }^{\mu_{3}} p_{1}^{2}\right. \\
& \left.-p_{1 \mu_{1}} p_{1}{ }^{\mu_{3}}\right) \widetilde{\Gamma}_{\mu_{3} \mu_{2}}\left(p_{1}, p_{3} ; p_{2}\right) \\
& +J\left(p_{2}^{2}\right) G\left(p_{3}^{2}\right)\left(g_{\mu_{2}}{ }^{\mu_{3}} p_{2}^{2}\right. \\
& \left.-p_{2 \mu_{2}} p_{2}{ }^{\mu_{3}}\right) \widetilde{\Gamma}_{\mu_{3} \mu_{1}}\left(p_{2}, p_{3} ; p_{1}\right)
\end{aligned}
$$

It is easy to see that the $F$ and $H$ functions from the threegluon vertex (2.4), as well as the $c$ and $e$ functions from the ghost-gluon vertex (2.10) do not contribute to this identity. Below, we are going to use Eq. (2.12) as a nontrivial check on the results for the longitudinal part of the three-gluon vertex.

To conclude this section, we would like to present the notation we use for the integrals occurring in the one-loop calculations. We define the integral corresponding to the triangle diagram as
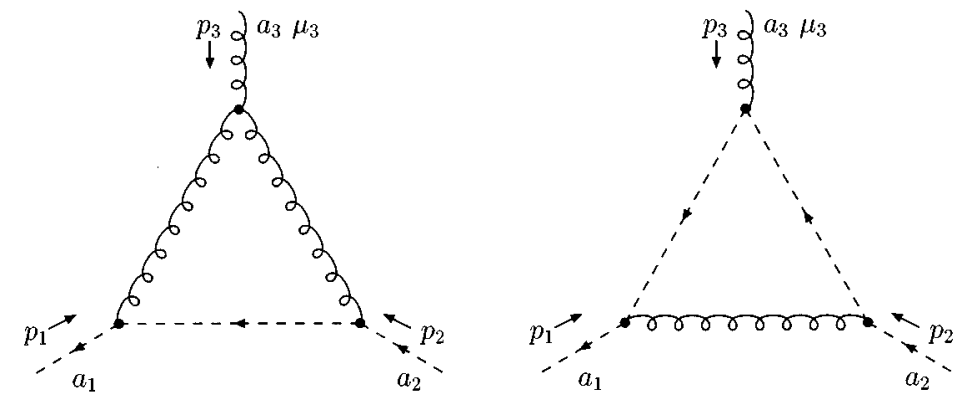

FIG. 3. One-loop ghost-gluon vertex diagrams. 


$$
J\left(\nu_{1}, \nu_{2}, \nu_{3}\right) \equiv \int \frac{d^{n} q}{\left[\left(p_{2}-q\right)^{2}\right]^{\nu_{1}}\left[\left(p_{1}+q\right)^{2}\right]^{\nu_{2}}\left(q^{2}\right)^{\nu_{3}}},
$$

where $n=4-2 \varepsilon$ is the space-time dimension (in the framework of dimensional regularization ${ }^{5}$ [23]). A brief overview of relevant results for such integrals in $n$ dimensions is presented in Appendix B. It should be noted that all such integrals occurring in the present calculation can be algebraically reduced to one nontrivial integral

$$
J(1,1,1)=i \pi^{n / 2} \eta \varphi\left(p_{1}^{2}, p_{2}^{2}, p_{3}^{2}\right),
$$

where $\varphi\left(p_{1}^{2}, p_{2}^{2}, p_{3}^{2}\right) \equiv \varphi$ is a totally symmetric function (see Appendix B for details), and three two-point integrals $J(0,1,1), J(1,0,1)$, and $J(1,1,0)$, which can be expressed in terms of a powerlike function

$$
\begin{aligned}
\kappa\left(p_{i}^{2}\right) & \equiv \kappa_{i}=-\frac{2}{(n-3)(n-4)}\left(-p_{i}^{2}\right)^{(n-4) / 2} \\
& =\frac{1}{\varepsilon(1-2 \varepsilon)}\left(-p_{i}^{2}\right)^{-\varepsilon}
\end{aligned}
$$

as, e.g.,

$$
J(1,1,0)=i \pi^{n / 2} \eta \kappa\left(p_{3}^{2}\right),
$$

and similarly for $J(0,1,1)$ and $J(1,0,1)$, where, instead of $\kappa\left(p_{3}^{2}\right)=\kappa_{3}$, we should use $\kappa\left(p_{1}^{2}\right)=\kappa_{1}$ and $\kappa\left(p_{2}^{2}\right)=\kappa_{2}$, respectively. In Eqs. (2.14) and (2.16), $\eta$ denotes a factor constructed of $\Gamma$ functions,

$$
\eta \equiv \frac{\Gamma^{2}(n / 2-1)}{\Gamma(n-3)} \Gamma\left(3-\frac{n}{2}\right)=\frac{\Gamma^{2}(1-\varepsilon)}{\Gamma(1-2 \varepsilon)} \Gamma(1+\varepsilon) .
$$

\section{OFF-SHELL RESULTS}

The set of Feynman diagrams yielding one-loop contributions to the three-gluon vertex is presented in Fig. 1.

When calculating the diagrams, we used the standard technique of tensor decomposition ${ }^{6}$ [24], reducing the result to combinations of scalar integrals multiplying the tensor structures constructed from the external momenta (see Ap- pendix A). In the Feynman gauge, the basic set of scalar integrals (2.13) includes the four integrals $J(1,1,1)$, $J(0,1,1), J(1,0,1)$, and $J(1,1,0)$ only, since massless integrals with two nonpositive powers $\nu_{i}$ vanish in dimensional regularization [23]. For arbitrary $\xi$, we also get integrals with some of the powers of the denominators equal to 2; see Eq. (2.3). However, with the help of the integration-by-parts technique [27] these integrals can be algebraically reduced to the above basic set (see Ref. [28]). While performing the calculations, the REDUCE system [29] was heavily employed.

Before presenting the results, let us define two totally symmetric combinations of the invariants formed from the external momenta:

$$
\mathcal{Q} \equiv\left(p_{1} p_{2}\right)+\left(p_{1} p_{3}\right)+\left(p_{2} p_{3}\right)=-\frac{1}{2}\left(p_{1}^{2}+p_{2}^{2}+p_{3}^{2}\right)
$$

$$
\begin{aligned}
\mathcal{K} & \equiv p_{1}^{2} p_{2}^{2}-\left(p_{1} p_{2}\right)^{2}=p_{1}^{2} p_{3}^{2}-\left(p_{1} p_{3}\right)^{2}=p_{2}^{2} p_{3}^{2}-\left(p_{2} p_{3}\right)^{2} \\
& =\left(p_{1} p_{2}\right)\left(p_{1} p_{3}\right)+\left(p_{1} p_{2}\right)\left(p_{2} p_{3}\right)+\left(p_{1} p_{3}\right)\left(p_{2} p_{3}\right) \\
& =-\frac{1}{4}\left[\left(p_{1}^{2}\right)^{2}+\left(p_{2}^{2}\right)^{2}+\left(p_{3}^{2}\right)^{2}-2 p_{1}^{2} p_{2}^{2}-2 p_{1}^{2} p_{3}^{2}-2 p_{2}^{2} p_{3}^{2}\right] .
\end{aligned}
$$

From the last line of Eq. (3.2), one can recognize the structure $-4 \mathcal{K}$ as the Källen function of $p_{1}^{2}, p_{2}^{2}$, and $p_{3}^{2}$; see, e.g., Ref. [30].

\section{A. Results in the Feynman gauge}

Let us consider the one-loop contributions to the threegluon vertex (2.4) in the Feynman gauge $(\xi=0)$, without the quark loops (the results for the latter are presented in Sec. III C). We shall use the standard notation $C_{A}$ for the Casimir constant,

$$
f^{a c d} f^{b c d}=C_{A} \delta^{a b} \quad\left[C_{A}=N \text { for the } \operatorname{SU}(N) \text { group }\right],
$$

whereas the factor $\eta$ occurring in the results is defined by Eq. (2.17).

The one-loop results for the scalar functions (2.4), for arbitrary value of the space-time dimension $n$, are

$$
\begin{aligned}
A^{(1,0)}\left(p_{1}^{2}, p_{2}^{2} ; p_{3}^{2}\right)= & \frac{g^{2} \eta}{(4 \pi)^{n / 2}} C_{A} \frac{1}{4(n-1) \mathcal{K}}\left\{(n-1)\left[p_{3}^{2}+3\left(p_{1} p_{2}\right)\right]\left[p_{3}^{2}\left(p_{1} p_{2}\right) \varphi+\left(p_{1} p_{3}\right) \kappa_{1}+\left(p_{2} p_{3}\right) \kappa_{2}+p_{3}^{2} \kappa_{3}\right]\right. \\
& \left.+4(n-1) \mathcal{K}\left[\left(p_{1} p_{2}\right) \varphi+\kappa_{3}\right]-(3 n-2) \mathcal{K}\left[\kappa_{1}+\kappa_{2}\right]\right\}, \\
B^{(1,0)}\left(p_{1}^{2}, p_{2}^{2} ; p_{3}^{2}\right)=- & \frac{g^{2} \eta}{(4 \pi)^{n / 2}} C_{A} \frac{1}{4(n-1) \mathcal{K}}\left(p_{1}^{2}-p_{2}^{2}\right)\left\{(n-1)\left[\left(p_{1} p_{3}\right)\left(p_{2} p_{3}\right) \varphi+\left(p_{1} p_{3}\right) \kappa_{1}+\left(p_{2} p_{3}\right) \kappa_{2}+p_{3}^{2} \kappa_{3}\right]\right.
\end{aligned}
$$

\footnotetext{
${ }^{5}$ For simplicity, we put the dimensional-regularization scale $\mu_{\mathrm{DR}}=1$. Otherwise, all one-loop expressions for dimensionally regularized quantities should have been multiplied by $\left(\mu_{\mathrm{DR}}\right)^{2 \varepsilon}$. In the final results, expanded around $n=4$ and renormalized, this scale can easily be restored by inserting $\mu_{\mathrm{DR}}$ in all nondimensionless arguments of the logarithms, in order to make them dimensionless. See also Sec. III E where the renormalization is discussed.
}

${ }^{6}$ An alternative way to decompose triangle integrals (2.13) with tensor numerators was used in [25]. It was based on a formula from [26]. 


$$
\begin{aligned}
& \left.+(4 n-3) \mathcal{K} \frac{\kappa_{1}-\kappa_{2}}{p_{1}^{2}-p_{2}^{2}}\right\}, \\
C^{(1,0)}\left(p_{1}^{2}, p_{2}^{2} ; p_{3}^{2}\right)= & \frac{g^{2} \eta}{(4 \pi)^{n / 2}} C_{A} \frac{1}{4(n-1) \mathcal{K}}\left\{3(n-1)\left[p_{3}^{2}\left(p_{1} p_{2}\right) \varphi+\left(p_{1} p_{3}\right) \kappa_{1}+\left(p_{2} p_{3}\right) \kappa_{2}+p_{3}^{2} \kappa_{3}\right]-2(4 n-3) \mathcal{K} \frac{\kappa_{1}-\kappa_{2}}{p_{1}^{2}-p_{2}^{2}}\right\}, \\
F^{(1,0)}\left(p_{1}^{2}, p_{2}^{2} ; p_{3}^{2}\right)= & \frac{g^{2} \eta}{(4 \pi)^{n / 2}} C_{A} \frac{1}{4(n-1) \mathcal{K}^{3}}\left\{2\left[\left(n^{2}-1\right)\left(p_{1} p_{2}\right)\left(p_{1} p_{3}\right)\left(p_{2} p_{3}\right)+2(n-2) p_{3}^{2} \mathcal{K}-(n-7)\left(p_{1} p_{2}\right) \mathcal{K}\right]\right. \\
& \times\left[p_{3}^{2}\left(p_{1} p_{2}\right) \varphi+\left(p_{1} p_{3}\right) \kappa_{1}+\left(p_{2} p_{3}\right) \kappa_{2}+p_{3}^{2} \kappa_{3}\right]+2 \mathcal{K}\left[(n+1)(n-4)\left(p_{1}^{2} p_{2}^{2}, p_{3}^{2}\right)=0\right. \\
& \times\left[\left(p_{1} p_{2}\right) \varphi+\kappa_{3}\right]+2 p_{3}^{2} \mathcal{K}\left[(n+1)\left(p_{1} p_{3}\right)\left(p_{2} p_{3}\right)+(n-3) \mathcal{K}\right] \varphi+(4 n-7) \mathcal{K}^{2}\left[\kappa_{1}+\kappa_{2}\right]+\mathcal{K}\{2(n+1) \mathcal{K}] \\
& \left.\left.\times\left(p_{1} p_{2}\right)\left(p_{1}^{2}-p_{2}^{2}\right)^{2}+(4 n-3) \mathcal{K}\left[p_{1}^{2}+p_{2}^{2}-2\left(p_{1} p_{2}\right)\right]\right\} \frac{\kappa_{1}-\kappa_{2}}{p_{1}^{2}-p_{2}^{2}}\right\} \\
& \left.\left.+p_{3}^{2}\left[p_{3}^{2}\left(p_{1} p_{2}\right)+\left(p_{1} p_{3}\right)\left(p_{2} p_{3}\right)\right] \kappa_{3}\right\}\right) . \\
H^{(1,0)}\left(p_{1}^{2}, p_{2}^{2}, p_{3}^{2}\right)= & \frac{g^{2} \eta}{(4 \pi)^{n / 2}} C_{A} \frac{1}{2(n-1) \mathcal{K}^{3}}\left(( n ^ { 2 } - 1 ) ( p _ { 1 } p _ { 2 } ) ( p _ { 1 } p _ { 3 } ) ( p _ { 2 } p _ { 3 } ) \left[\left(p_{1} p_{2}\right)\left(p_{1} p_{3}\right)\left(p_{2} p_{3}\right) \varphi+\left(p_{1} p_{2}\right)\left(p_{1} p_{3}\right) \kappa_{1}\right.\right. \\
& \left.+\left(p_{1} p_{2}\right)\left(p_{2} p_{3}\right) \kappa_{2}+\left(p_{1} p_{3}\right)\left(p_{2} p_{3}\right) \kappa_{3}\right]-3(n-1)\left(p_{1} p_{2}\right)\left(p_{1} p_{3}\right)\left(p_{2} p_{3}\right) \mathcal{K}\left[\mathcal{Q} \varphi+\kappa_{1}+\kappa_{2}+\kappa_{3}\right]
\end{aligned}
$$

When expanded around $n=4$, these formulas coincide ${ }^{7}$ with the results presented in [13]. We shall see that the result $S=0$ is valid also in an arbitrary gauge. It should be noted that presenting the results in arbitrary dimension does not spoil their compactness, as compared with the formulas expanded around $n=4$.

\section{B. Results in arbitrary covariant gauge}

In an arbitrary gauge, the results for the scalar functions of the three-gluon vertex (2.4) are obviously less compact than those in the Feynman gauge. We list them below, also for arbitrary value of the space-time dimension:

$$
\begin{aligned}
A^{(1, \xi)}\left(p_{1}^{2}, p_{2}^{2} ; p_{3}^{2}\right)= & \frac{g^{2} \eta}{(4 \pi)^{n / 2}} C_{A} \frac{1}{32 \mathcal{K}^{2} p_{1}^{2} p_{2}^{2}}\left[\left(p_{1}^{2} p_{2}^{2} \mathcal{K}\left\{\left[8-4 \xi-(n-2)(n-3) \xi^{2}\right] p_{3}^{2}+2\left[12+4(n-3) \xi+(n-3) \xi^{2}\right]\left(p_{1} p_{2}\right)\right\}\right.\right. \\
& \left.+\xi[(n-4) \xi+4] \mathcal{K} \mathcal{Q}\left[(n-3)\left(p_{1} p_{2}\right) \mathcal{Q}-(n-4) \mathcal{K}\right]+\xi[(n-3) \xi+2](n-1) p_{1}^{2} p_{2}^{2} p_{3}^{2}\left(p_{1} p_{2}\right) \mathcal{Q}\right)\left[p_{3}^{2}\left(p_{1} p_{2}\right) \varphi\right. \\
& \left.+\left(p_{1} p_{3}\right) \kappa_{1}+\left(p_{2} p_{3}\right) \kappa_{2}+p_{3}^{2} \kappa_{3}\right]-\mathcal{K}\left([ ( n - 4 ) \xi + 4 ] \mathcal { K } \left\{[(n-4) \xi-8] p_{1}^{2} p_{2}^{2}+\xi \mathcal{Q}\left[(n-2) p_{3}^{2}-2(n-3)\right.\right.\right. \\
& \left.\left.\left.\times\left(p_{1} p_{2}\right)\right]\right\}-\xi[(n-3) \xi+2](n-2) p_{1}^{2} p_{2}^{2} p_{3}^{2} \mathcal{Q}\right)\left[\left(p_{1} p_{2}\right) \varphi+\kappa_{3}\right]+\mathcal{K} \varphi\left(\xi [ ( n - 4 ) \xi + 4 ] \mathcal { K } \left\{(2 n-7) p_{1}^{2} p_{2}^{2} p_{3}^{2}\right.\right. \\
& \left.\left.+\mathcal{Q}\left[p_{1}^{2}\left(p_{1} p_{3}\right)+p_{2}^{2}\left(p_{2} p_{3}\right)\right]\right\}+\xi[(n-3) \xi+2] p_{1}^{2} p_{2}^{2} p_{3}^{2}\left[p_{3}^{2} \mathcal{Q}-2(n-4) \mathcal{K}\right]\right)-\frac{\mathcal{K}}{n-1}\left\{p_{1}^{2} p_{2}^{2} \mathcal{K}[8(3 n-2)\right. \\
& \left.+4(n-1)(5 n-17) \xi-3(n-1)(n-4) \xi^{2}\right]+\xi[(n-4) \xi+4](n-1) \mathcal{K} \mathcal{Q}^{2} \\
& \left.\left.+\xi[(n-3) \xi+2](n-1) p_{1}^{2} p_{2}^{2} p_{3}^{2} \mathcal{Q}\right\}\left[\kappa_{1}+\kappa_{2}\right]\right]
\end{aligned}
$$

${ }^{7} \mathrm{Up}$ to the definition of the renormalization scheme constant $C$ in [13], which we find to be $C=-\gamma-\ln \pi+2$ rather than $C=-\gamma-\ln \pi$. 


$$
\begin{aligned}
& B^{(1, \xi)}\left(p_{1}^{2}, p_{2}^{2} ; p_{3}^{2}\right)=\frac{g^{2} \eta}{(4 \pi)^{n / 2}} C_{A} \frac{1}{32 \mathcal{K}^{2} p_{1}^{2} p_{2}^{2} p_{3}^{2}}\left(p_{1}^{2}-p_{2}^{2}\right)\left[\left(-p_{1}^{2} p_{2}^{2} p_{3}^{2} \mathcal{K}\left[8-12 \xi-(n+2)(n-3) \xi^{2}\right]+\xi[(n-4) \xi+4] \mathcal{K} \mathcal{Q}\{(n\right.\right. \\
& \left.\left.-2) \mathcal{K}+(n-3)\left(p_{1} p_{2}\right)\left[p_{3}^{2}+\left(p_{1} p_{2}\right)\right]\right\}+\xi[(n-3) \xi+2] p_{1}^{2} p_{2}^{2}\left\{2 \mathcal{K} \mathcal{Q}+(n-1) p_{3}^{2}\left(p_{1} p_{2}\right)\left[p_{3}^{2}+\left(p_{1} p_{2}\right)\right]\right\}\right) \\
& \times\left[p_{3}^{2}\left(p_{1} p_{2}\right) \varphi+\left(p_{1} p_{3}\right) \kappa_{1}+\left(p_{2} p_{3}\right) \kappa_{2}+p_{3}^{2} \kappa_{3}\right]+(n-4) \mathcal{K}\left\{\xi[(n-4) \xi+4] \mathcal{K}\left(p_{3}^{2} \mathcal{Q}+p_{1}^{2} p_{2}^{2}\right)+\xi[(n-3) \xi\right. \\
& \left.+2] p_{1}^{2} p_{2}^{2} p_{3}^{2}\left[p_{3}^{2}+\left(p_{1} p_{2}\right)\right]\right\}-\mathcal{K} p_{3}^{2} \varphi\left\{p_{1}^{2} p_{2}^{2} \mathcal{K}\left[8+4(n-5) \xi-(3 n-10) \xi^{2}\right]+\xi[(n-4) \xi+4] \mathcal{K} \mathcal{Q}^{2}-\xi[(n\right. \\
& \left.-3) \xi+2] p_{1}^{2} p_{2}^{2} p_{3}^{2}\left[p_{3}^{2}+\left(p_{1} p_{2}\right)\right]\right\}-\frac{\mathcal{K}}{n-1} \frac{\kappa_{1}-\kappa_{2}}{p_{1}^{2}-p_{2}^{2}}\left\{p_{1}^{2} p_{2}^{2} p_{3}^{2} \mathcal{K}[8(4 n-3)+4(n-1)(5 n-19) \xi-(n-1)\right. \\
& \left.\times(5 n-18) \xi^{2}\right]+\xi[(n-4) \xi+4](n-1) \mathcal{K} \mathcal{Q}\left[p_{1}^{2}\left(p_{1} p_{3}\right)+p_{2}^{2}\left(p_{2} p_{3}\right)\right] \\
& \left.\left.-\xi[(n-3) \xi+2](n-1) p_{1}^{2} p_{2}^{2}\left(p_{1}^{2}-p_{2}^{2}\right)^{2}\left[p_{3}^{2}+\left(p_{1} p_{2}\right)\right]\right\}\right], \\
& C^{(1, \xi)}\left(p_{1}^{2}, p_{2}^{2} ; p_{3}^{2}\right)=\frac{g^{2} \eta}{(4 \pi)^{n / 2}} C_{A} \frac{1}{16 \mathcal{K}^{2} p_{1}^{2} p_{2}^{2} p_{3}^{2}}\left(\left\{2 p_{1}^{2} p_{2}^{2} p_{3}^{2} \mathcal{K}\left[6+(2 n-5) \xi+(n-3) \xi^{2}\right]+\xi[(n-4) \xi+4]\right.\right. \\
& \left.\times \mathcal{Q K}\left[\mathcal{K}+(n-3) p_{3}^{2}\left(p_{1} p_{2}\right)\right]+\xi[(n-3) \xi+2](n-1) p_{1}^{2} p_{2}^{2}\left(p_{3}^{2}\right)^{2}\left(p_{1} p_{2}\right)\right\}\left[p_{3}^{2}\left(p_{1} p_{2}\right) \varphi+\left(p_{1} p_{3}\right) \kappa_{1}\right. \\
& \left.+\left(p_{2} p_{3}\right) \kappa_{2}+p_{3}^{2} \kappa_{3}\right]+(n-4) \mathcal{K} p_{3}^{2}\left\{\xi[(n-4) \xi+4] \mathcal{K} \mathcal{Q}+\xi[(n-3) \xi+2] p_{1}^{2} p_{2}^{2} p_{3}^{2}\right\}\left[\left(p_{1} p_{2}\right) \varphi+\kappa_{3}\right] \\
& +\mathcal{K} p_{3}^{2} \varphi\left\{\xi[(n-4) \xi+4] \mathcal{K}\left(p_{1}^{2} p_{2}^{2}-\mathcal{Q}^{2}\right)+\xi[(n-3) \xi+2] p_{1}^{2} p_{2}^{2}\left(p_{3}^{2}\right)^{2}\right\}-\frac{\mathcal{K}}{n-1} \frac{\kappa_{1}-\kappa_{2}}{p_{1}^{2}-p_{2}^{2}} \\
& \times\left\{2 p_{1}^{2} p_{2}^{2} p_{3}^{2} \mathcal{K}\left[4(4 n-3)+2(n-1)(5 n-18) \xi-(n-1)(2 n-7) \xi^{2}\right]+\xi[(n-4) \xi+4](n-1)\right. \\
& \left.\left.\times \mathcal{K} \mathcal{Q}\left[p_{1}^{2}\left(p_{1} p_{3}\right)+p_{2}^{2}\left(p_{2} p_{3}\right)\right]-\xi[(n-3) \xi+2](n-1) p_{1}^{2} p_{2}^{2} p_{3}^{2}\left(p_{1}^{2}-p_{2}^{2}\right)^{2}\right\}\right), \\
& S^{(1, \xi)}\left(p_{1}^{2}, p_{2}^{2}, p_{3}^{2}\right)=0,
\end{aligned}
$$

$$
\begin{aligned}
& F^{(1, \xi)}\left(p_{1}^{2}, p_{2}^{2} ; p_{3}^{2}\right)=\frac{g^{2} \eta}{(4 \pi)^{n / 2}} C_{A} \frac{1}{32(n-1) \mathcal{K}^{3} p_{1}^{2} p_{2}^{2} p_{3}^{2}}\left\{2 \left(p _ { 1 } ^ { 2 } p _ { 2 } ^ { 2 } p _ { 3 } ^ { 2 } \left\{\left(p_{1} p_{2}\right)\left(p_{1} p_{3}\right)\left(p_{2} p_{3}\right)(n-1)[8(n+1)+8(n-3) \xi\right.\right.\right. \\
& \left.+\left(3 n^{2}-38 n+63\right) \xi^{2}-(n-3)(7 n-13) \xi^{3}\right]-2 \mathcal{K} \mathcal{Q}\left[8(n-2)+8(n-1) \xi+(n-1)(3 n-14) \xi^{2}\right. \\
& \left.\left.-2(n-1)(n-3) \xi^{3}\right]+\mathcal{K}\left(p_{1} p_{2}\right)\left[8(n+3)-(n-1)\left(n^{2}-4 n+23\right) \xi^{2}-7(n-1)(n-3) \xi^{3}\right]\right\} \\
& -\xi[(n-4) \xi+4](n-1)\left\{[(n-3) \xi+2](n-3)\left(p_{1} p_{2}\right)^{2}\left(p_{1} p_{3}\right)^{2}\left(p_{2} p_{3}\right)^{2}+[(n-3) \xi+n-1]\right. \\
& \left.\left.\times \mathcal{K}\left(p_{1} p_{2}\right)^{2}\left(p_{1} p_{3}\right)\left(p_{2} p_{3}\right)-[(n-3) \xi+3 n-7] \mathcal{K}^{2} p_{1}^{2} p_{2}^{2}+3(n-4) \mathcal{K}^{3}\right\}\right)\left[p_{3}^{2}\left(p_{1} p_{2}\right) \varphi+\left(p_{1} p_{3}\right) \kappa_{1}\right. \\
& \left.+\left(p_{2} p_{3}\right) \kappa_{2}+p_{3}^{2} \kappa_{3}\right]+2 \mathcal{K}\left(p _ { 1 } ^ { 2 } p _ { 2 } ^ { 2 } p _ { 3 } ^ { 2 } \left\{( p _ { 1 } p _ { 3 } ) ( p _ { 2 } p _ { 3 } ) ( n - 4 ) \left[8(n+1)+8(n-1) \xi+(n-1)(3 n-23) \xi^{2}\right.\right.\right. \\
& \left.-5(n-1)(n-3) \xi^{3}\right]-\mathcal{K}\left[8(5 n-11)+4(n-1)(5 n-11) \xi+(n-1)\left(n^{2}+4 n-10\right) \xi^{2}+n(n-1)\right. \\
& \left.\left.\times(n-3) \xi^{3}\right]\right\}-\xi[(n-4) \xi+4](n-1)(n-4)\left\{[(n-3) \xi+2]\left(p_{1} p_{2}\right)\left(p_{1} p_{3}\right)^{2}\left(p_{2} p_{3}\right)^{2}+\mathcal{K}\left(p_{1} p_{2}\right)\left(p_{1} p_{3}\right)\right. \\
& \left.\left.\times\left(p_{2} p_{3}\right)-3 \mathcal{K}^{2}\left(p_{1} p_{2}\right)\right\}\right)\left[\left(p_{1} p_{2}\right) \varphi+\kappa_{3}\right]+2 \mathcal{K} \varphi\left(p _ { 1 } ^ { 2 } p _ { 2 } ^ { 2 } ( p _ { 3 } ^ { 2 } ) ^ { 2 } \left\{\mathcal { K } ( n - 1 ) \left[16+16 \xi+(5 n-32) \xi^{2}-6\right.\right.\right. \\
& \left.\left.\times(n-3) \xi^{3}\right]+p_{3}^{2}\left(p_{1} p_{2}\right)\left[8(n+1)+8(n-1) \xi+(n-1)(3 n-23) \xi^{2}-5(n-1)(n-3) \xi^{3}\right]\right\}-\xi[(n-4) \xi \\
& +4](n-1)\left(p_{1} p_{3}\right)\left(p_{2} p_{3}\right)\left\{[(n-3) \xi+2] p_{3}^{2}\left(p_{1} p_{2}\right)\left(p_{1} p_{3}\right)\left(p_{2} p_{3}\right)+[(n-3) \xi+3] \mathcal{K} p_{3}^{2}\left(p_{1} p_{2}\right)\right. \\
& \left.\left.+\xi(n-4) \mathcal{K}^{2}\right\}\right)+\mathcal{K}^{2} p_{1}^{2} p_{2}^{2} p_{3}^{2}\left[8(4 n-7)+4(n-1)(5 n-11) \xi+(n-1)(13 n-30) \xi^{2}+2(n-1)(n-2)\right. \\
& \left.\times(n-3) \xi^{3}\right]\left[\kappa_{1}+\kappa_{2}\right]+\mathcal{K} \frac{\kappa_{1}-\kappa_{2}}{p_{1}^{2}-p_{2}^{2}}\left[p _ { 1 } ^ { 2 } p _ { 2 } ^ { 2 } p _ { 3 } ^ { 2 } \left(\mathcal{K}\left[p_{3}^{2}-4\left(p_{1} p_{2}\right)\right][8(4 n-3)+4(n-1)(5 n-17) \xi\right.\right. \\
& \left.\left.-(n-1)(3 n-10) \xi^{2}\right]+2\left(p_{1} p_{2}\right)\left(p_{1}^{2}-p_{2}^{2}\right)^{2}(n+1)\left\{8-(n-1) \xi^{2}[(n-3) \xi+3]\right\}\right) \\
& \left.\left.-2 \xi[(n-4) \xi+4](n-1) \mathcal{K}\left\{\mathcal{K}\left[p_{1}^{2}\left(p_{1} p_{3}\right)+p_{2}^{2}\left(p_{2} p_{3}\right)\right]-[(n-3) \xi+2]\left(p_{1} p_{3}\right)\left(p_{2} p_{3}\right)\left(p_{1}^{2}-p_{2}^{2}\right)^{2}\right\}\right]\right\},
\end{aligned}
$$




$$
\begin{aligned}
H^{(1, \xi)}\left(p_{1}^{2}, p_{2}^{2}, p_{3}^{2}\right)= & \frac{g^{2} \eta}{(4 \pi)^{n / 2}} C_{A} \frac{1}{16 \mathcal{K}^{3} p_{1}^{2} p_{2}^{2} p_{3}^{2}}\left[\left(p _ { 1 } ^ { 2 } p _ { 2 } ^ { 2 } p _ { 3 } ^ { 2 } ( p _ { 1 } p _ { 2 } ) ( p _ { 1 } p _ { 3 } ) ( p _ { 2 } p _ { 3 } ) \left[8(n+1)+14(n-1) \xi+4(n-1)(n-7) \xi^{2}\right.\right.\right. \\
& \left.-5(n-1)(n-3) \xi^{3}\right]-\xi[(n-4) \xi+4]\left\{(n-1)[(n-3) \xi+3]\left(p_{1} p_{2}\right)^{2}\left(p_{1} p_{3}\right)^{2}\left(p_{2} p_{3}\right)^{2}+3(n-4) \mathcal{K}^{3}\right\} \\
& \left.-(n-1) \xi(2-\xi)\left(p_{1}^{2}\right)^{2}\left(p_{2}^{2}\right)^{2}\left(p_{3}^{2}\right)^{2}\right)\left[\left(p_{1} p_{2}\right)\left(p_{1} p_{3}\right)\left(p_{2} p_{3}\right) \varphi+\left(p_{1} p_{2}\right)\left(p_{1} p_{3}\right) \kappa_{1}+\left(p_{1} p_{2}\right)\left(p_{2} p_{3}\right) \kappa_{2}\right. \\
& \left.+\left(p_{1} p_{3}\right)\left(p_{2} p_{3}\right) \kappa_{3}\right]+\mathcal{K}\left\{-p_{1}^{2} p_{2}^{2} p_{3}^{2}\left(p_{1} p_{2}\right)\left(p_{1} p_{3}\right)\left(p_{2} p_{3}\right)\left[24+38 \xi+4(3 n-16) \xi^{2}-9(n-3) \xi^{3}\right]\right. \\
& \left.+3 \xi[(n-4) \xi+4][(n-3) \xi+3]\left(p_{1} p_{2}\right)^{2}\left(p_{1} p_{3}\right)^{2}\left(p_{2} p_{3}\right)^{2}+\xi(2-\xi)\left(p_{1}^{2}\right)^{2}\left(p_{2}^{2}\right)^{2}\left(p_{3}^{2}\right)^{2}\right\}\left[\mathcal{Q} \varphi+\kappa_{1}+\kappa_{2}+\kappa_{3}\right] \\
& +\mathcal{K}^{3} \varphi\left\{p_{1}^{2} p_{2}^{2} p_{3}^{2}\left[16+4(3 n-8) \xi-(n-2) \xi^{2}-(n-2) \xi^{3}\right]-\xi^{2}[(n-4) \xi+4](n-4)\left(p_{1} p_{2}\right)\left(p_{1} p_{3}\right)\left(p_{2} p_{3}\right)\right\} \\
& +\frac{\mathcal{K}}{n-1}\left\{2 p_{1}^{2} p_{2}^{2} p_{3}^{2}\left[4(n-2)+6(n-1) \xi+(n-1)(2 n-9) \xi^{2}-(n-1)(n-3) \xi^{3}\right]-\xi[(n-4) \xi+4]\right. \\
& \left.\times[(n-3) \xi+3](n-1)\left(p_{1} p_{2}\right)\left(p_{1} p_{3}\right)\left(p_{2} p_{3}\right)\right\}\left\{p_{1}^{2}\left[p_{1}^{2}\left(p_{2} p_{3}\right)+\left(p_{1} p_{2}\right)\left(p_{1} p_{3}\right)\right] \kappa_{1}+p_{2}^{2}\left[p_{2}^{2}\left(p_{1} p_{3}\right)+\left(p_{1} p_{2}\right)\right.\right. \\
& \left.\left.\left.\times\left(p_{2} p_{3}\right)\right] \kappa_{2}+p_{3}^{2}\left[p_{3}^{2}\left(p_{1} p_{2}\right)+\left(p_{1} p_{3}\right)\left(p_{2} p_{3}\right)\right] \kappa_{3}\right\}\right] .
\end{aligned}
$$

One of the main technical problems we met in this calculation was how to bring the results for arbitrary $\xi$ to a reasonably short form. The original REDUCE output for the numerators of the scalar functions (2.4) was really huge. Then, the problem was how to organize the result and which bases to choose. The first basis we needed was one in the "space" of the scalar functions $\varphi$ and $\kappa_{i}$. It was possible to get a better factorization of the coefficients by considering not these functions themselves but certain linear combinations. Moreover, not all "convenient" combinations happened to be the same for the different functions; see Eqs. (3.10)(3.15). Then, the coefficients multiplying these combinations of $\varphi$ and $\kappa_{i}$ are polynomials in $\xi, n$, and the momentum invariants. Trying to write the latter only as $p_{1}^{2}, p_{2}^{2}$, and $p_{3}^{2}$, we were still getting rather long expressions. The next idea was to try to use in some cases also the scalar products $\left(p_{1} p_{2}\right),\left(p_{1} p_{3}\right)$, and $\left(p_{2} p_{3}\right)$, together with the notation (3.1) and (3.2) for symmetric combinations. These tricks (as well as looking for proper combinations of $\xi$ and $n$ ) allowed us to write the expressions in a much shorter form. However, this part of the work could not be completely automatized since $p_{i}^{2}$ and the scalar products $\left(p_{i} p_{j}\right)$ are linearly dependent.

There are some special values of the gauge parameter $\xi$ we would like to point out. First of all, we see that the terms containing $p_{1}^{2}, p_{2}^{2}$, or $p_{3}^{2}$ in the denominator disappear not only if we put $\xi=0$ (Feynman gauge), but also in a "singular" (in four dimensions) gauge, ${ }^{8} \xi=-4 /(n-4)$. Having no $p_{i}^{2}$ in the denominator is especially convenient when one considers on-shell limits, i.e., when some of the external momenta squared vanish; otherwise, one needs to expand the scalar integrals in the vanishing momenta squared (see Sec. IV). Second, many terms vanish for $\xi=-2 /(n-3)$, which could be considered an $n$-dimensional generalization of the Fried-Yennie gauge [31] (see also Ref. [32] and Appendix D).

\section{Contributions of the quark loops}

Let us consider the quark loop contributions to the functions (2.4). We assume that there are $N_{f}$ quarks which are all massless, and we define

$$
T_{R}=\frac{1}{8} \operatorname{Tr}(I)=\frac{1}{2}[\text { if } \operatorname{Tr}(I)=4],
$$

where $I$ is the "unity" in the space of Dirac matrices.

The quark loop contributions do not depend on $\xi$. The results of the calculation are

$$
\begin{aligned}
A^{(1, q)}\left(p_{1}^{2}, p_{2}^{2} ; p_{3}^{2}\right) & =\frac{g^{2} \eta}{(4 \pi)^{n / 2}} N_{f} T_{R} \frac{n-2}{n-1}\left[\kappa_{1}+\kappa_{2}\right], \\
B^{(1, q)}\left(p_{1}^{2}, p_{2}^{2} ; p_{3}^{2}\right) & =\frac{g^{2} \eta}{(4 \pi)^{n / 2}} N_{f} T_{R} \frac{n-2}{n-1}\left[\kappa_{1}-\kappa_{2}\right], \\
C^{(1, q)}\left(p_{1}^{2}, p_{2}^{2} ; p_{3}^{2}\right) & =\frac{g^{2} \eta}{(4 \pi)^{n / 2}} 2 N_{f} T_{R} \frac{n-2}{n-1} \frac{\kappa_{1}-\kappa_{2}}{p_{1}^{2}-p_{2}^{2}},
\end{aligned}
$$

\footnotetext{
${ }^{8}$ It is not clear whether the second choice could be of use in realistic calculations, since a singularity of $\xi$ in four dimensions requires extra care in renormalizing, etc.
} 


$$
\begin{aligned}
& S^{(1, q)}\left(p_{1}^{2}, p_{2}^{2}, p_{3}^{2}\right)=0 \\
& F^{(1, q)}\left(p_{1}^{2}, p_{2}^{2} ; p_{3}^{2}\right)=-\frac{g^{2} \eta}{(4 \pi)^{n / 2}} N_{f} T_{R} \frac{1}{(n-1)(n-2) \mathcal{K}^{3}}\left(2\left[\left(n^{2}-1\right)\left(p_{1} p_{2}\right)\left(p_{1} p_{3}\right)\left(p_{2} p_{3}\right)+2(n-2) p_{3}^{2} \mathcal{K}-(n-7)\left(p_{1} p_{2}\right) \mathcal{K}\right]\right. \\
& \times\left[p_{3}^{2}\left(p_{1} p_{2}\right) \varphi+\left(p_{1} p_{3}\right) \kappa_{1}+\left(p_{2} p_{3}\right) \kappa_{2}+p_{3}^{2} \kappa_{3}\right]+2(n+1)(n-4) \mathcal{K} p_{3}^{2}\left(p_{1} p_{2}\right)\left[\left(p_{1} p_{2}\right) \varphi+\kappa_{3}\right] \\
&+2 p_{3}^{2} \mathcal{K} \varphi\left[(n+1)\left(p_{1} p_{3}\right)\left(p_{2} p_{3}\right)+(n-3) \mathcal{K}\right]+n(n-4) \mathcal{K}^{2}\left[\kappa_{1}+\kappa_{2}\right]+\mathcal{K} \frac{\kappa_{1}-\kappa_{2}}{p_{1}^{2}-p_{2}^{2}} \\
&\left.\times\left\{2(n+1)\left(p_{1} p_{2}\right)\left(p_{1}^{2}-p_{2}^{2}\right)^{2}+(n-2)^{2} \mathcal{K}\left[p_{1}^{2}+p_{2}^{2}-2\left(p_{1} p_{2}\right)\right]\right\}\right), \\
& H^{(1, q)}\left(p_{1}^{2}, p_{2}^{2}, p_{3}^{2}\right)=-\frac{g^{2} \eta}{(4 \pi)^{n / 2}} 2 N_{f} T_{R} \frac{1}{(n-1)(n-2) \mathcal{K}^{3}}\left(( n ^ { 2 } - 1 ) ( p _ { 1 } p _ { 2 } ) ( p _ { 1 } p _ { 3 } ) ( p _ { 2 } p _ { 3 } ) \left[\left(p_{1} p_{2}\right)\left(p_{1} p_{3}\right)\left(p_{2} p_{3}\right) \varphi+\left(p_{1} p_{2}\right)\right.\right. \\
&\left.\times\left(p_{1} p_{3}\right) \kappa_{1}+\left(p_{1} p_{2}\right)\left(p_{2} p_{3}\right) \kappa_{2}+\left(p_{1} p_{3}\right)\left(p_{2} p_{3}\right) \kappa_{3}\right]-3(n-1)\left(p_{1} p_{2}\right)\left(p_{1} p_{3}\right)\left(p_{2} p_{3}\right) \mathcal{K}\left[\mathcal{Q} \varphi+\kappa_{1}+\kappa_{2}+\kappa_{3}\right] \\
&+(n-1)(n-2) \mathcal{K}^{3} \varphi+(n-2) \mathcal{K}\left\{p_{1}^{2}\left[p_{1}^{2}\left(p_{2} p_{3}\right)+\left(p_{1} p_{2}\right)\left(p_{1} p_{3}\right)\right] \kappa_{1}+p_{2}^{2}\left[p_{2}^{2}\left(p_{1} p_{3}\right)+\left(p_{1} p_{2}\right)\left(p_{2} p_{3}\right)\right] \kappa_{2}\right. \\
&\left.\left.+p_{3}^{2}\left[p_{3}^{2}\left(p_{1} p_{2}\right)+\left(p_{1} p_{3}\right)\left(p_{2} p_{3}\right)\right] \kappa_{3}\right\}\right) .
\end{aligned}
$$

\section{Symmetric limit and comparison}

Now, we would like to compare to our results with those by Celmaster and Gonsalves [11]. For their study of renormalization-prescription dependence of Green functions, they evaluated the three-gluon vertex function to one loop at the symmetric point:

$$
p_{1}^{2}=p_{2}^{2}=p_{3}^{2} \equiv p^{2}=-M^{2} .
$$

In this case, we get $\left(p_{1} p_{2}\right)=\left(p_{1} p_{3}\right)=\left(p_{2} p_{3}\right)=-\frac{1}{2} p^{2}=\frac{1}{2} M^{2}$. At the symmetric point, the vertex function simplifies considerably. First of all, because of their antisymmetry, the $B$ and $S$ functions (2.4) must be zero,

$$
B\left(p^{2}, p^{2} ; p^{2}\right)=S\left(p^{2}, p^{2}, p^{2}\right) \equiv 0 .
$$

Furthermore, in this limit, the number of independent tensor combinations in the three-gluon vertex reduces to 3 , and the vertex function can be written, in the notation used in [11], as ${ }^{9}$

$$
\begin{aligned}
\Gamma_{\mu_{1} \mu_{2} \mu_{3}}\left(p_{1}, p_{2}, p_{3}\right)= & G_{0}\left(p^{2}\right)\left[g_{\mu_{1} \mu_{2}}\left(p_{1}-p_{2}\right)_{\mu_{3}}+g_{\mu_{2} \mu_{3}}\left(p_{2}-p_{3}\right)_{\mu_{1}}+g_{\mu_{3} \mu_{1}}\left(p_{3}-p_{1}\right)_{\mu_{2}}\right]-G_{1}\left(p^{2}\right)\left(p_{2}-p_{3}\right)_{\mu_{1}}\left(p_{3}-p_{1}\right)_{\mu_{2}}\left(p_{1}\right. \\
& \left.-p_{2}\right)_{\mu_{3}}+G_{2}\left(p^{2}\right)\left(p_{1 \mu_{3}} p_{2 \mu_{1}} p_{3 \mu_{2}}-p_{1 \mu_{2}} p_{2 \mu_{3}} p_{3 \mu_{1}}\right),
\end{aligned}
$$

with the three $G_{i}$ functions related to the scalar functions in Eq. (2.4) through

$$
\begin{gathered}
G_{0}\left(p^{2}\right)=A\left(p^{2}, p^{2} ; p^{2}\right)+\frac{1}{2} p^{2} C\left(p^{2}, p^{2} ; p^{2}\right)+\frac{1}{4}\left(p^{2}\right)^{2} F\left(p^{2}, p^{2} ; p^{2}\right)+\frac{1}{2} p^{2} H\left(p^{2}, p^{2}, p^{2}\right), \\
G_{1}\left(p^{2}\right)=C\left(p^{2}, p^{2} ; p^{2}\right)+\frac{1}{2} p^{2} F\left(p^{2}, p^{2} ; p^{2}\right), \\
G_{2}\left(p^{2}\right)=C\left(p^{2}, p^{2} ; p^{2}\right)+\frac{1}{2} p^{2} F\left(p^{2}, p^{2} ; p^{2}\right)+H\left(p^{2}, p^{2}, p^{2}\right) .
\end{gathered}
$$

We note that two of these relations may be expressed more compactly as

$$
G_{2}\left(p^{2}\right)=G_{1}\left(p^{2}\right)+H\left(p^{2}, p^{2}, p^{2}\right), \quad G_{0}\left(p^{2}\right)=A\left(p^{2}, p^{2} ; p^{2}\right)+\frac{1}{2} p^{2} G_{2}\left(p^{2}\right) .
$$

From our results, we obtain the following expressions for the $G_{i}$ functions (in arbitrary gauge and dimension):

$$
\begin{aligned}
G_{0}^{(1, \xi)}\left(p^{2}\right)= & \frac{g^{2} \eta}{(4 \pi)^{n / 2}} C_{A} \frac{1}{288}\left\{p^{2} \varphi\left[8+12 \xi(14 n-51)+6 \xi^{2}\left(n^{2}-18 n+60\right)-\xi^{3}(n-4)(n-12)\right]-6 \kappa[32+36 \xi(2 n-7)\right. \\
& \left.\left.+6 \xi^{2}(n-4)(n-6)-\xi^{3}(n-4)(n-3)\right]\right\},
\end{aligned}
$$

${ }^{9}$ The counterterm contribution is omitted. 


$$
\begin{gathered}
G_{1}^{(1, \xi)}\left(p^{2}\right)=-\frac{g^{2} \eta}{(4 \pi)^{n / 2}} C_{A} \frac{1}{3456 p^{2}}\left\{p^{2} \varphi\left[64(n-20)-144 \xi(7 n-26)-24 \xi^{2}\left(n^{2}-18 n+50\right)+\xi^{3}\left(n^{3}-24 n^{2}+200 n-384\right)\right]\right. \\
\left.-6 \kappa \frac{n-4}{n-1}\left[64(n-2)-144 \xi(n-1)-12 \xi^{2}(n-1)(2 n-7)+\xi^{3}(n-1)(n-3)(n-20)\right]\right\}, \\
G_{2}^{(1, \xi)}\left(p^{2}\right)=\frac{g^{2} \eta}{(4 \pi)^{n / 2}} C_{A} \frac{1}{144 p^{2}}\left\{p^{2} \varphi\left[128+6 \xi(29 n-114)+6 \xi^{2}\left(n^{2}-18 n+60\right)-\xi^{3}(n-4)(n-12)\right]\right. \\
\left.-6 \kappa \frac{n-4}{n-1}\left[8+30 \xi(n-1)+6 \xi^{2}(n-1)(n-5)-\xi^{3}(n-1)(n-3)\right]\right\}, \\
G_{0}^{(1, q)}\left(p^{2}\right)=-\frac{g^{2} \eta}{(4 \pi)^{n / 2}} N_{f} T_{R} \frac{2(3 n-8)}{9(n-2)}\left\{2 p^{2} \varphi-3 \kappa\right\}, \\
G_{1}^{(1, q)}\left(p^{2}\right)=-\frac{g^{2} \eta}{(4 \pi)^{n / 2}} N_{f} T_{R} \frac{4}{27 p^{2}}\left\{4 p^{2} \varphi+3 \kappa \frac{n-4}{n-1}\right\}, \\
G_{2}^{(1, q)}\left(p^{2}\right)=-\frac{g^{2} \eta}{(4 \pi)^{n / 2}} N_{f} T_{R} \frac{4}{9(n-2) p^{2}}\left\{2 p^{2} \varphi(3 n-8)-3 \kappa \frac{n-4}{n-1}\right\}
\end{gathered}
$$

where $\varphi=\varphi\left(p^{2}, p^{2}, p^{2}\right)$ and $\kappa=\kappa\left(p^{2}\right)$. Expanding these results around $n=4$ and keeping the divergent and finite (in $\varepsilon=(4-n) / 2)$ terms only, we arrive at exactly the same results as Celmaster and Gonsalves; see Eqs. (14a), (14b), and (14c) of [11]. ${ }^{10}$

In Ref. [33] the QCD renormalization has been considered at an asymmetric point:

$$
p_{1}^{2}=p_{2}^{2} \equiv p^{2}=-M^{2}, \quad p_{3}^{2}=4 z p^{2}=-4 z M^{2}
$$

In particular, the three-gluon vertex was studied at this point, including quark loop contributions (with massive quarks). The tensor structures used to decompose the threegluon vertex are presented in Eqs. (2) and (4) of [33]. Of the scalar functions multiplying the seven tensor structures defined by Eq. (5) of [33], an explicit result is presented for the function $F_{0}$ only; see Eq. (6) of [33]. ${ }^{11}$ In terms of the scalar functions (2.4), one finds the correspondence $F_{0} \leftrightarrow-A\left(p^{2}, 4 z p^{2} ; p^{2}\right)-B\left(p^{2}, 4 z p^{2} ; p^{2}\right)$.

Calculating this combination of the $A$ and $B$ functions for the case (3.36), we find coincidence of the $\xi$ and $\xi^{2}$

${ }^{10}$ Their transcendental constant $I$ is nothing but our $\left.p^{2} \varphi\left(p^{2}, p^{2}, p^{2}\right)\right|_{n=4}$ [see Eq. (B10)] which can be expressed in terms of Clausen's function as $(4 / \sqrt{3}) \mathrm{Cl}_{2}(\pi / 3)$.

${ }^{11} \mathrm{We}$ note some misprints in [33]: (i) In the definition of the $\varphi$ function [Eq. (8)], $x$ in the denominator of the expression under the square root should read $z$; (ii) before Eq. (7), the definition of $1 / \hat{\varepsilon}$ should read $1 / \hat{\varepsilon}=2 /(D-4)+\gamma+\ln \left[Q^{2} /\left(4 \pi \nu^{2}\right)\right]$ (the sign before the logarithm should be changed). contributions, ${ }^{12}$ as well as the quark loop contibutions in the massless limit. However, our result for the Feynman-gauge part is different. So we do not confirm Eq. (6) of [33].

\section{E. Renormalization}

In the limit $n \rightarrow 4(\varepsilon \rightarrow 0)$, the only function which may have an ultraviolet singularity is the $A$ function, since this is the only function which does not vanish at the "zero-loop" level; see Eq. (2.6). In arbitrary gauge, the ultravioletsingular part of the $A$ function follows from Eq. (3.10),

$$
A^{(1, \mathrm{UV})}=\frac{g^{2} \eta}{(4 \pi)^{2-\varepsilon}}\left[-C_{A}\left(\frac{2}{3}+\frac{3}{4} \xi\right)+\frac{4}{3} N_{f} T_{R}\right] \kappa^{(\mathrm{UV})},
$$

where $\kappa^{(\mathrm{UV})}=1 / \varepsilon+\cdots$ is the ultraviolet-singular part of the $\kappa$ function (2.15). Therefore, the divergent part of the counterterm contribution should be equal to minus the RHS of Eq. (3.37). This counterterm contribution can be written as ${ }^{13}$

$$
\begin{aligned}
A^{(1, \mathrm{CT})} & =\frac{\vec{g}^{2}}{(4 \pi)^{2}}\left[C_{A}\left(\frac{2}{3}+\frac{3}{4} \xi\right)-\frac{3}{4} N_{f} T_{R}\right]\left(\frac{1}{\varepsilon}+R\right) \\
& =\frac{g^{2} \eta}{(4 \pi)^{2-\varepsilon}}\left[C_{A}\left(\frac{2}{3}+\frac{3}{4} \xi\right)-\frac{3}{4} N_{f} T_{R}\right]\left(\frac{1}{\varepsilon}+R\right)+O(\varepsilon),
\end{aligned}
$$

\footnotetext{
${ }^{12}$ In [33], $b$ is the same as our $\xi$. The integral $I(0, z)$ from [33] is related, in the limit (3.36), to our $J(1,1,1)$ as $I(0, z)=-\left(i \pi^{2}\right)^{-1} M^{2} J(1,1,1)$.

${ }^{13}$ In commonly used notation, expression (3.38) corresponds to $\left(Z_{1}-1\right)$ at the one-loop order.
} 
where $R$ is a constant corresponding to the choice of renormalization scheme, whereas $\vec{g}^{2} \equiv g^{2} e^{-\gamma \varepsilon}(4 \pi)^{\varepsilon}$ $=g^{2} \exp \{\varepsilon[-\gamma+\ln (4 \pi)]\}$ is the "rescaled" coupling constant. Such a redefinition of $g^{2}$ is usually performed in the context of the modified minimal subtraction $(\overline{\mathrm{MS}})$ renormalization scheme [34] which corresponds to the choice $R=0$ [for $R=0$, Eq. (3.38) corresponds to Eq. (15) of Ref. [11]]. The second line of Eq. (3.38) is more convenient for dealing with the expressions obtained in the present paper, since one can keep $g^{2} \eta /(4 \pi)^{n / 2}$ as an overall factor. Here, we have used the fact that

$$
\eta=e^{-\gamma \varepsilon}\left[1-\frac{1}{12} \pi^{2} \varepsilon^{2}+O\left(\varepsilon^{3}\right)\right]
$$

The $\varepsilon^{2}$ term in Eq. (3.39) is not relevant for the ultraviolet renormalization at the one-loop level. However, it yields finite contributions when one has $1 / \varepsilon^{2}$ infrared (on-shell) singularities (see Sec. IV C).

If we now recall the existence of the dimensionalregularization scale parameter $\mu_{\mathrm{DR}}$ (which we usually put equal to 1; see footnote at the end of Sec. II), we shall see that, as opposed to other one-loop-order contributions, the counterterm (3.38) should not be multiplied by the factor $\left(\mu_{\mathrm{DR}}\right)^{2 \varepsilon}$; see also in Ref. [11].

Examining Eq. (3.37), it is interesting to note that for

$$
\left.\xi\right|_{n=4} \equiv \xi_{0}=\frac{8}{9}\left(\frac{2 N_{f} T_{R}}{C_{A}}-1\right)
$$

we do not have any ultraviolet singularity in the one-loop contribution to the three-gluon vertex. If we substitute $N_{f}=6, \quad C_{A}=3$, and $T_{R}=\frac{1}{2}$, we get $\xi_{0}=\frac{8}{9}$.

\section{ON-SHELL LIMITS}

There are two main on-shell cases of interest: when one or two of the external momenta squared are zero. However, it is also instructive to consider, as a separate case, the limit when one external momentum (not only its square) vanishes.

\section{A. One external momentum squared is zero, $p_{3}^{2}=0$}

In this case, we should substitute in the expressions for all scalar functions

$$
\begin{gathered}
p_{3}^{2}=0, \quad\left(p_{1} p_{2}\right)=-\frac{1}{2}\left(p_{1}^{2}+p_{2}^{2}\right), \\
\left(p_{1} p_{3}\right)=-\frac{1}{2}\left(p_{1}^{2}-p_{2}^{2}\right), \\
\left(p_{2} p_{3}\right)=\frac{1}{2}\left(p_{1}^{2}-p_{2}^{2}\right) .
\end{gathered}
$$

Note that now we should consider the scalar functions $A, B, C$, and $F$ from Eq. (2.4) with permuted arguments as well.

The result for the triangle integral (2.14) simplifies in this limit:

$$
\begin{aligned}
\left.J(1,1,1)\right|_{p_{3}^{2}=0} & =i \pi^{2-\varepsilon} \eta \varphi\left(p_{1}^{2}, p_{2}^{2}, 0\right) \\
& =i \pi^{2-\varepsilon} \eta \frac{1}{\varepsilon^{2}} \frac{\left(-p_{1}^{2}\right)^{-\varepsilon}-\left(-p_{2}^{2}\right)^{-\varepsilon}}{p_{1}^{2}-p_{2}^{2}}
\end{aligned}
$$

where $\eta$ is defined by Eq. (2.17). Moreover, in the framework of dimensional regularization [23],

$$
\left.J(1,1,0)\right|_{p_{3}^{2}=0}=0,
$$

while the results for $J(1,0,1)$ and $J(0,1,1)$ remain unchanged. As to the factor $1 / \varepsilon^{2}$ in Eq. (4.2), one power of $\varepsilon$ is canceled by the expansion of the numerator, while another power of $\varepsilon$ survives and corresponds to the infrared (onshell) singularity which arises in the scalar integral in the limit $p_{3}^{2}=0$.

For the Feynman gauge $\xi=0$ [and also for the singular gauge $\xi=-4 /(n-4)]$, it is enough to perform the above substitutions to get the answer. In the case of arbitrary $\xi$, however, the situation is more tricky, due to the presence of $p_{3}^{2}$ in the denominators of the scalar functions. Here, in order to get a correct answer, one needs to expand the integral $J(1,1,1)$ in $p_{3}^{2}$ and keep the term of order $p_{3}^{2}$ :

$$
\begin{aligned}
\left.J(1,1,1)\right|_{p_{3}^{2} \rightarrow 0}= & i \pi^{2-\varepsilon} \eta \frac{1}{\varepsilon^{2}}\left\{\frac{\left(-p_{1}^{2}\right)^{-\varepsilon}-\left(-p_{2}^{2}\right)^{-\varepsilon}}{p_{1}^{2}-p_{2}^{2}}-\frac{p_{3}^{2}}{(1+\varepsilon)\left(p_{1}^{2}-p_{2}^{2}\right)^{2}}\right. \\
& \left.\times\left[(1-\varepsilon)\left[\left(-p_{1}^{2}\right)^{-\varepsilon}+\left(-p_{2}^{2}\right)^{-\varepsilon}\right]+2 \frac{\left(-p_{1}^{2}\right)^{1-\varepsilon}-\left(-p_{2}^{2}\right)^{1-\varepsilon}}{p_{1}^{2}-p_{2}^{2}}\right]\right\}+O\left(\left(p_{3}^{2}\right)^{2}\right) .
\end{aligned}
$$


To present the results obtained for the scalar functions (2.4) in this limit, it is convenient to introduce

$$
\delta_{12} \equiv \frac{p_{1}^{2}-p_{2}^{2}}{p_{1}^{2}+p_{2}^{2}} .
$$

In this section, we present such results for the three-gluon scalar functions in the Feynman gauge, and also for the quark loop contributions. The expressions for an arbitrary covariant gauge are listed in Appendix E. We present the results for the $A, B, C$, and $F$ functions of the arguments $\left(p_{1}^{2}, p_{2}^{2} ; 0\right)$ and $\left(0, p_{1}^{2} ; p_{2}^{2}\right)$. The results for the third set of arguments corresponding to the cyclic permutations in Eq. (2.4), $\left(p_{2}^{2}, 0 ; p_{1}^{2}\right)$, can be obtained from the functions of $\left(0, p_{1}^{2} ; p_{2}^{2}\right)$ by using the symmetry (for the $A, C$, and $F$ functions) or antisymmetry (for the $B$ function) with respect to the first two arguments, and interchanging $p_{1}^{2} \leftrightarrow p_{2}^{2}$. The $H$ function with permuted arguments does not change, while the $S$ function is zero (at one loop).

The results for the gluon and ghost contributions to the three-gluon scalar functions in the Feynman gauge are

$$
\begin{aligned}
& A^{(1,0)}\left(p_{1}^{2}, p_{2}^{2} ; 0\right)=-\frac{g^{2} \eta}{(4 \pi)^{n / 2}} C_{A} \frac{1}{4(n-1)(n-4)}\left\{(n-4)(3 n-2)\left[\kappa_{1}+\kappa_{2}\right]-n(n-1)\left(\delta_{12}\right)^{-1}\left[\kappa_{1}-\kappa_{2}\right]\right\}, \\
& A^{(1,0)}\left(0, p_{1}^{2} ; p_{2}^{2}\right)=-\frac{g^{2} \eta}{(4 \pi)^{n / 2}} C_{A} \frac{1}{4(n-1)(n-4)\left(p_{1}^{2}-p_{2}^{2}\right)}\left\{(n-4) \kappa_{1}\left[(2 n-1) p_{1}^{2}-(4 n-3) p_{2}^{2}\right]-2(n-1)\left(\delta_{12}\right)^{-1}\left[\kappa_{1}-\kappa_{2}\right]\right. \\
& \left.\times\left(2 p_{1}^{2}-3 p_{2}^{2}\right)\right\}, \\
& B^{(1,0)}\left(p_{1}^{2}, p_{2}^{2} ; 0\right)=-\frac{g^{2} \eta}{(4 \pi)^{n / 2}} C_{A} \frac{1}{4(n-1)(n-4)}\left(4 n^{2}-21 n+14\right)\left[\kappa_{1}-\kappa_{2}\right], \\
& B^{(1,0)}\left(0, p_{1}^{2} ; p_{2}^{2}\right)=-\frac{g^{2} \eta}{(4 \pi)^{n / 2}} C_{A} \frac{1}{4(n-1)(n-4)\left(p_{1}^{2}-p_{2}^{2}\right)^{2}}\left\{-(n-4)\left(p_{1}^{2}-p_{2}^{2}\right) \kappa_{1}\left[(6 n-5) p_{1}^{2}-(4 n-3) p_{2}^{2}\right]\right. \\
& \left.+2(n-1) p_{1}^{2}\left[\kappa_{1}-\kappa_{2}\right]\left[(n-3) p_{1}^{2}-(n-5) p_{2}^{2}\right]\right\} \text {, } \\
& C^{(1,0)}\left(p_{1}^{2}, p_{2}^{2} ; 0\right)=-\frac{g^{2} \eta}{(4 \pi)^{n / 2}} C_{A} \frac{1}{2(n-1)\left(p_{1}^{2}-p_{2}^{2}\right)} n\left[\kappa_{1}-\kappa_{2}\right], \\
& C^{(1,0)}\left(0, p_{1}^{2} ; p_{2}^{2}\right)=-\frac{g^{2} \eta}{(4 \pi)^{n / 2}} C_{A} \frac{1}{2(n-1)(n-4) p_{1}^{2}\left(p_{1}^{2}-p_{2}^{2}\right)^{2}}\left\{(n-4)\left(p_{1}^{2}-p_{2}^{2}\right) \kappa_{1}\left[n p_{1}^{2}-(4 n-3) p_{2}^{2}\right]\right. \\
& \left.+6(n-1) p_{1}^{2} p_{2}^{2}\left[\kappa_{1}-\kappa_{2}\right]\right\} \text {, } \\
& F^{(1,0)}\left(p_{1}^{2}, p_{2}^{2} ; 0\right)=-\frac{g^{2} \eta}{(4 \pi)^{n / 2}} C_{A} \frac{1}{(n-1)(n-4)\left(p_{1}^{2}-p_{2}^{2}\right)^{2}}(4 n-7)\left\{(n-4)\left[\kappa_{1}+\kappa_{2}\right]-2\left(\delta_{12}\right)^{-1}\left[\kappa_{1}-\kappa_{2}\right]\right\}, \\
& F^{(1,0)}\left(0, p_{1}^{2} ; p_{2}^{2}\right)=-\frac{g^{2} \eta}{(4 \pi)^{n / 2}} C_{A} \frac{1}{(n-1)(n-4) p_{1}^{2}\left(p_{1}^{2}-p_{2}^{2}\right)^{2}}\left\{(n-4)\left(p_{1}^{2}-p_{2}^{2}\right) \kappa_{1}\left[(3 n+1)-(n+2)\left(\delta_{12}\right)^{-1}-6\left(\delta_{12}\right)^{-2}\right]\right. \\
& \left.+2 p_{1}^{2}\left[\kappa_{1}-\kappa_{2}\right]\left[\left(n^{2}-12 n+17\right)+4(n-4)\left(\delta_{12}\right)^{-1}+6\left(\delta_{12}\right)^{-2}\right]\right\}, \\
& H^{(1,0)}\left(p_{1}^{2}, p_{2}^{2} ; 0\right)=-\frac{g^{2} \eta}{(4 \pi)^{n / 2}} C_{A} \frac{1}{(n-1)(n-4)\left(p_{1}^{2}-p_{2}^{2}\right)}\left\{3(n-4)\left(\delta_{12}\right)^{-1}\left[\kappa_{1}+\kappa_{2}\right]+\left[\kappa_{1}-\kappa_{2}\right]\left[\left(n^{2}-2 n-2\right)-6\left(\delta_{12}\right)^{-2}\right]\right\} .
\end{aligned}
$$

The quark loop contributions in the limit $p_{3}^{2}=0$ are

$$
\begin{gathered}
A^{(1, q)}\left(p_{1}^{2}, p_{2}^{2} ; 0\right)=\frac{g^{2} \eta}{(4 \pi)^{n / 2}} N_{f} T_{R} \frac{n-2}{n-1}\left[\kappa_{1}+\kappa_{2}\right], \\
A^{(1, q)}\left(0, p_{1}^{2} ; p_{2}^{2}\right)=\frac{g^{2} \eta}{(4 \pi)^{n / 2}} N_{f} T_{R} \frac{n-2}{n-1} \kappa_{1}, \\
B^{(1, q)}\left(p_{1}^{2}, p_{2}^{2} ; 0\right)=\frac{g^{2} \eta}{(4 \pi)^{n / 2}} N_{f} T_{R} \frac{n-2}{n-1}\left[\kappa_{1}-\kappa_{2}\right],
\end{gathered}
$$




$$
\begin{aligned}
& B^{(1, q)}\left(0, p_{1}^{2} ; p_{2}^{2}\right)=-\frac{g^{2} \eta}{(4 \pi)^{n / 2}} N_{f} T_{R} \frac{n-2}{n-1} \kappa_{1}, \\
& C^{(1, q)}\left(p_{1}^{2}, p_{2}^{2} ; 0\right)=\frac{g^{2} \eta}{(4 \pi)^{n / 2}} 2 N_{f} T_{R} \frac{n-2}{n-1} \frac{\kappa_{1}-\kappa_{2}}{p_{1}^{2}-p_{2}^{2}} \\
& C^{(1, q)}\left(0, p_{1}^{2} ; p_{2}^{2}\right)=\frac{g^{2} \eta}{(4 \pi)^{n / 2}} 2 N_{f} T_{R} \frac{n-2}{n-1} \frac{\kappa_{1}}{p_{1}^{2}} \\
& F^{(1, q)}\left(p_{1}^{2}, p_{2}^{2} ; 0\right)=\frac{g^{2} \eta}{(4 \pi)^{n / 2}} 4 N_{f} T_{R} \frac{n}{(n-1)(n-2)\left(p_{1}^{2}-p_{2}^{2}\right)^{2}}\left\{(n-4)\left[\kappa_{1}+\kappa_{2}\right]-2\left(\delta_{12}\right)^{-1}\left[\kappa_{1}-\kappa_{2}\right]\right\}, \\
& F^{(1, q)}\left(0, p_{1}^{2} ; p_{2}^{2}\right)=\frac{g^{2} \eta}{(4 \pi)^{n / 2}} 4 N_{f} T_{R} \frac{1}{(n-1)(n-2)(n-4) p_{1}^{2}\left(p_{1}^{2}-p_{2}^{2}\right)}\left\{(n-4) \kappa_{1}\left[\left(n^{2}-5 n+8\right)-\left(\delta_{12}\right)^{-1}(n+2)-6\left(\delta_{12}\right)^{-2}\right]\right. \\
& \left.+16 \frac{p_{1}^{2} p_{2}^{2}}{\left(p_{1}^{2}-p_{2}^{2}\right)^{3}}\left[\kappa_{1}-\kappa_{2}\right]\left[(n-1) p_{1}^{2}-(n-4) p_{2}^{2}\right]\right\} \text {, } \\
& H^{(1, q)}\left(p_{1}^{2}, p_{2}^{2} ; 0\right)=\frac{g^{2} \eta}{(4 \pi)^{n / 2}} 4 N_{f} T_{R} \frac{1}{(n-1)(n-2)(n-4)\left(p_{1}^{2}-p_{2}^{2}\right)}\left\{3(n-4)\left(\delta_{12}\right)^{-1}\left[\kappa_{1}+\kappa_{2}\right]+\left[(n-2)\left(n^{2}-5 n+7\right)\right.\right. \\
& \left.\left.-6\left(\delta_{12}\right)^{-2}\right]\left[\kappa_{1}-\kappa_{2}\right]\right\} \text {. }
\end{aligned}
$$

We note that there is an interesting relation between the one-loop contributions to the $A$ and $B$ functions of permuted arguments: ${ }^{14}$

$$
\left(p_{1}^{2}+p_{2}^{2}\right) A^{(1)}\left(p_{1}^{2}, p_{2}^{2} ; 0\right)-p_{1}^{2} A^{(1)}\left(0, p_{1}^{2} ; p_{2}^{2}\right)-p_{2}^{2} A^{(1)}\left(0, p_{2}^{2} ; p_{1}^{2}\right)+p_{1}^{2} B^{(1)}\left(0, p_{2}^{2} ; p_{1}^{2}\right)+p_{2}^{2} B^{(1)}\left(0, p_{1}^{2} ; p_{2}^{2}\right)=0
$$

This relation is also satisfied by the expressions for arbitrary $\xi$ given in Appendix E.

The infrared $1 / \varepsilon$ singularities of the results for gluon and ghost contributions (also in arbitrary gauge; see Appendix E) have been compared with the results given in [14], Eqs. (24)-(25). The functions $G^{j}$ defined in [14] are proportional to our functions $Z_{i j k}$ (see Appendix A) which can be represented as linear combinations of the scalar functions (2.4), including those with permuted arguments. To get renormalized results, the counterterm (3.38) was added to all $A$ functions. In the $\overline{\mathrm{MS}}$ scheme, the obtained results coincide ${ }^{15}$ with those presented in Ref. [14], Eq. (25).

\section{B. One external momentum is zero, $p_{3}=0$}

In this case, $p_{1}=-p_{2} \equiv p\left(p_{1}^{2}=p_{2}^{2}=p^{2}\right)$, and the proper limit of Eq. (4.2) yields

$$
\left.J(1,1,1)\right|_{p_{3}=0}=i \pi^{2-\varepsilon} \eta \frac{1}{\varepsilon}\left(-p^{2}\right)^{-1-\varepsilon} .
$$

Actually, we get some powers of $\left(p_{1}^{2}-p_{2}^{2}\right)$ in the denominator from the $\mathcal{K}$ 's, Eq. (3.2), since $\mathcal{K}=-\frac{1}{4}\left(p_{1}^{2}-p_{2}^{2}\right)^{2}$ in this limit. Therefore, we should be careful taking the limit $p_{2}^{2} \rightarrow p_{1}^{2}$ and expand the numerator up to higher powers of

$$
\delta_{12}^{\prime} \equiv \frac{p_{1}^{2}-p_{2}^{2}}{p_{1}^{2}}=\frac{2 \delta_{12}}{1+\delta_{12}} .
$$

Starting from the result for the case $p_{3}^{2}=0$, we need to express $\kappa_{2}$ as $\kappa_{1}$ times an expansion in $\delta_{12}^{\prime}$. This can easily be done using

$$
J(1,0,1)=J(0,1,1)\left(\frac{p_{2}^{2}}{p_{1}^{2}}\right)^{-\varepsilon}=J(0,1,1) \sum_{j=0}^{\infty} \frac{(\varepsilon)_{j}}{j !}\left(\delta_{12}^{\prime}\right)^{j}
$$

\footnotetext{
${ }^{14}$ This is also valid for the zero-loop functions, since $A^{(0)}=1$ and $B^{(0)}=0$.

${ }^{15} \mathrm{Up}$ to a misprint in Eq. (25g) of [14] where, in the term proportional to $\lambda$ (their $\lambda$ is nothing but our $\xi$ ), the contribution $l\left(1+11 / 4+2 f^{2}\right)$ should read $l\left(1+11 / 4 f+2 f^{2}\right)$.
} 
In practice, we need the terms up to $\left(\delta_{12}^{\prime}\right)^{3}$ only. It is interesting that in this special case we do not need the $p_{3}^{2}$ term of the expansion of $J(1,1,1)$ [like in Eq. (4.4)] since it cancels in all contributions.

In this limit, there are only three independent tensor structures left, and the coefficients multiplying these structures can be expressed in terms of the "surviving" scalar functions as ${ }^{16}$

$$
\begin{aligned}
\Gamma_{\mu_{1} \mu_{2} \mu_{3}}(p,-p, 0)= & 2 g_{\mu_{1} \mu_{2}} p_{\mu_{3}}\left[A\left(p^{2}, p^{2} ; 0\right)+p^{2} C\left(p^{2}, p^{2} ; 0\right)\right]-\left(g_{\mu_{1} \mu_{3}} p_{\mu_{2}}+g_{\mu_{2} \mu_{3}} p_{\mu_{1}}\right)\left[A\left(0, p^{2} ; p^{2}\right)-B\left(0, p^{2} ; p^{2}\right)\right] \\
& -2 p_{\mu_{1}} p_{\mu_{2}} p_{\mu_{3}} C\left(p^{2}, p^{2} ; 0\right) .
\end{aligned}
$$

The one-loop contributions to the scalar functions appearing in Eq. (4.28) are

$$
\begin{gathered}
{\left[A^{(1, \xi)}\left(p^{2}, p^{2} ; 0\right)+p^{2} C^{(1, \xi)}\left(p^{2}, p^{2} ; 0\right)\right]=} \\
-\frac{g^{2} \eta}{(4 \pi)^{n / 2}} \frac{C_{A} \kappa}{32(n-1)}\left\{8(3 n-4)+12 \xi(n-1)\left(n^{2}-4 n+2\right)\right. \\
\left.-\xi^{2}(n-1)(n-4)(n+2)\right\}, \\
{\left[A^{(1, \xi)}\left(0, p^{2} ; p^{2}\right)-B^{(1, \xi)}\left(0, p^{2} ; p^{2}\right)\right]=A^{(1, \xi)}\left(p^{2}, p^{2} ; 0\right)=\frac{g^{2} \eta}{(4 \pi)^{n / 2}} \frac{C_{A} \kappa}{8(n-1)}\left\{2\left(n^{2}-7 n+4\right)-2 \xi(n-1)(4 n-13)\right.} \\
\left.+\xi^{2}(n-1)(n-4)\right\}, \\
C^{(1, \xi)}\left(p^{2}, p^{2} ; 0\right)=-\frac{g^{2} \eta}{(4 \pi)^{n / 2}} \frac{C_{A}(n-4) \kappa}{32(n-1) p^{2}}\left\{8 n+4 \xi(n-1)(3 n-8)-\xi^{2}(n-1)(n-2)\right\}, \\
{\left[A^{(1, q)}\left(p^{2}, p^{2} ; 0\right)+p^{2} C^{(1, q)}\left(p^{2}, p^{2} ; 0\right)\right]=\frac{g^{2} \eta}{(4 \pi)^{n / 2}} N_{f} T_{R} \frac{(n-2)^{2}}{n-1} \kappa,} \\
{\left[A^{(1, q)}\left(0, p^{2} ; p^{2}\right)-B^{(1, q)}\left(0, p^{2} ; p^{2}\right)\right]=A^{(1, q)}\left(p^{2}, p^{2} ; 0\right)=\frac{g^{2} \eta}{(4 \pi)^{n / 2}} 2 N_{f} T_{R} \frac{n-2}{n-1} \kappa,} \\
C^{(1, q)}\left(p^{2}, p^{2} ; 0\right)=\frac{g^{2} \eta}{(4 \pi)^{n / 2}} N_{f} T_{R} \frac{(n-4)(n-2)}{n-1} \frac{\kappa}{p^{2}},
\end{gathered}
$$

where $\kappa \equiv \kappa\left(p^{2}\right)$.

We note that, according to Eqs. (4.30) and (4.33), the following relation holds for the zero-momentum case:

$$
A^{(1)}\left(p^{2}, p^{2} ; 0\right)-A^{(1)}\left(0, p^{2} ; p^{2}\right)+B^{(1)}\left(0, p^{2} ; p^{2}\right)=0 .
$$

This also follows from Eq. (4.24). The relation (4.35) is valid for arbitrary values of $n$ and $\xi$. Using Eq. (4.35), we can reduce the number of tensor structures in Eq. (4.28) from 3 to 2 : namely, ${ }^{17}$

$$
\Gamma_{\mu_{1} \mu_{2} \mu_{3}}^{(1)}(p,-p, 0)=\left(2 g_{\mu_{1} \mu_{2}} p_{\mu_{3}}-g_{\mu_{1} \mu_{3}} p_{\mu_{2}}-g_{\mu_{2} \mu_{3}} p_{\mu_{1}}\right) A^{(1)}\left(p^{2}, p^{2} ; 0\right)+2 p_{\mu_{3}}\left(p^{2} g_{\mu_{1} \mu_{2}}-p_{\mu_{1}} p_{\mu_{2}}\right) C^{(1)}\left(p^{2}, p^{2} ; 0\right),
$$

where the results for the scalar functions are given in Eqs. (4.30), (4.31), (4.33), and (4.34). Note that the first tensor structure on the RHS of Eq. (4.36) coincides with the "zeroloop' vertex structure, given by Eq. (2.1).

To make the complete comparison of our expressions (4.29)-(4.31) [contributing to the three-gluon vertex at $p_{3}=0$, Eq. (4.28)] with Eq. (20) of [14], we need to renormalize our coefficients (4.29) and (4.30) by adding the counterterm (3.38) to all $A$ functions involved and putting $n=4$. Performing this in the $\overline{\mathrm{MS}}$ scheme (i.e., at $R=0$ ), we find that our results give the same as Eq. (20) of [14].

${ }^{16}$ This corresponds to the decomposition used in Ref. [14], Eq. (20).
We have also compared the renormalized (in the $\overline{\mathrm{MS}}$ scheme) version of Eq. (4.36) with the one-loop results presented in Ref. [35], Eq. (A10). According to Eq. (4.36) there should be the following correspondence between the functions $T_{1}$ and $T_{2}$ used in [35] and $A^{(1)}$ and $C^{(1)}$ :

$$
T_{1}\left(p^{2}\right) \leftrightarrow A^{(1)}\left(p^{2}, p^{2} ; 0\right), \quad T_{2}\left(p^{2}\right) \leftrightarrow-2 p^{2} C^{(1)}\left(p^{2}, p^{2} ; 0\right)
$$

Renormalizing $A^{(1)}\left(p^{2}, p^{2} ; 0\right)$ [given by the sum of Eqs. (4.30) and (4.33) $]$ and putting $n=4$, we arrive at the same

\footnotetext{
${ }^{17}$ This corresponds to the decomposition used in Ref. [35], Eq. (A2).
} 
result for $T_{1}$ as given in Eq. (A10) of Ref. [35]. ${ }^{18}$ The result for $T_{2}$ is finite as $n \rightarrow 4$ and should correspond to the sum of our Eqs. (4.31) and (4.34) taken at $n=4$. However, our result for $T_{2}$ is different from the one given in Eq. (A10) of [35]. ${ }^{19}$

\section{Two external momenta squared are zero, $p_{1}^{2}=p_{2}^{2}=0$}

In this case, we substitute

$$
\begin{gathered}
p_{1}^{2}=p_{2}^{2}=0, \quad p_{3}^{2} \equiv p^{2}, \quad\left(p_{1} p_{2}\right)=\frac{1}{2} p^{2}, \quad\left(p_{1} p_{3}\right)=\left(p_{2} p_{3}\right) \\
=-\frac{1}{2} p^{2}, \\
\left.J(1,1,1)\right|_{p_{1}^{2}=p_{2}^{2}=0}=i \pi^{2-\varepsilon} \eta \varphi\left(0,0, p^{2}\right) \\
=-i \pi^{2-\varepsilon} \eta \frac{1}{\varepsilon^{2}}\left(-p^{2}\right)^{-1-\varepsilon} \\
\left.J(1,0,1)\right|_{p_{1}^{2}=p_{2}^{2}=0}=\left.J(0,1,1)\right|_{p_{1}^{2}=p_{2}^{2}=0}=0,
\end{gathered}
$$

while the result for $J(1,1,0)$ remains unchanged, Eq. (2.16). Note that now, when two external lines are on shell, the infrared singularity in $J(1,1,1)$ is stronger and gives $1 / \varepsilon^{2}$, Eq. (4.39).

Again, it is enough to make the above substitutions to get the result in the Feynman gauge $(\xi=0)$ and in the singular gauge $[\xi=-4 /(n-4)]$, but the situation is more tricky for arbitrary $\xi$ since we have $p_{1}^{2}$ and $p_{2}^{2}$ in the denominators of the scalar functions. To solve this problem, we need to consider the expansion of $J(1,1,1)$ in $p_{1}^{2}$ and $p_{2}^{2}$.

Two independent ways were used to get the results for the scalar functions in this limit.

(i) We take the expressions for one of the momenta squared equal to zero (see Sec. IV A), and put the second momentum squared equal to zero. In the corresponding expressions (see Appendix E), all $p_{i}^{2}$ occurring in the denominators are always accompanied by the corresponding $\kappa_{i} \equiv \kappa\left(p_{i}^{2}\right)$ in the numerator, which should be put equal to zero when $p_{i}^{2}$ vanishes.

(ii) First, we put $J(1,0,1)=J(0,1,1)=0$. Since the resulting expressions did not have singularities for $p_{1}^{2}=p_{2}^{2}$, the next step was to put $p_{1}^{2}=p_{2}^{2} \equiv p_{0}^{2}$. Then, the integral $J(1,1,1)$ was expanded in $p_{0}^{2} / p^{2}$, keeping the terms up to $\left(p_{0}^{2} / p^{2}\right)^{2}$. And, finally, the limit $p_{0}^{2} \rightarrow 0$ was taken. The following formula was used to expand $J(1,1,1)$ :

$$
\begin{aligned}
& \left.J(1,1,1)\right|_{p_{1}^{2}=p_{2}^{2} \equiv p_{0}^{2}}=-\frac{i \pi^{2-\varepsilon}}{\left(-p^{2}\right)^{1+\varepsilon}} \eta \frac{1}{\varepsilon^{2}}{ }_{2} F_{1}\left(\begin{array}{c|c}
1, \frac{1}{2}+\varepsilon & \frac{4 p_{0}^{2}}{p^{2}} \\
1+2 \varepsilon
\end{array}\right), \\
& { }_{2} F_{1}\left(\begin{array}{c|c}
1, \frac{1}{2}+\varepsilon \\
1+2 \varepsilon
\end{array} \mid \frac{4 p_{0}^{2}}{p^{2}}\right)=\sum_{j=0}^{\infty}\left(\frac{4 p_{0}^{2}}{p^{2}}\right)^{j} \frac{\left(\frac{1}{2}+\varepsilon\right)_{j}}{(1+2 \varepsilon)_{j}}=1+2 \frac{p_{0}^{2}}{p^{2}} \\
& +4 \frac{n-7}{n-6}\left(\frac{p_{0}^{2}}{p^{2}}\right)^{2}+\cdots
\end{aligned}
$$

The results obtained in these two ways coincide, and the expressions obtained for the scalar functions (2.4) are presented below. Because of the symmetry properties, the functions $A, C$, and $F$ of the arguments $\left(0, p^{2}, 0\right)$ are equal to the corresponding functions of the arguments $\left(p^{2}, 0,0\right)$, while the $B$ function with these arguments permuted changes sign. The $H$ function is the same for all permutations.

The resulting one-loop contributions (without quark loops) to the scalar functions (2.4) in arbitrary gauge are

$$
\begin{gathered}
A^{(1, \xi)}\left(0,0 ; p^{2}\right)=\frac{g^{2} \eta}{(4 \pi)^{n / 2}} C_{A} \frac{1}{32(n-4)} \kappa\left\{48-8 \xi(n-3)(n-6)+\xi^{2}(n-4)^{2}\right\} \\
A^{(1, \xi)}\left(p^{2}, 0 ; 0\right)=-\frac{g^{2} \eta}{(4 \pi)^{n / 2}} C_{A} \frac{1}{64(n-1)(n-4)} \kappa\left\{16\left(2 n^{2}-13 n+8\right)+4 \xi(n-1)(2 n-9)(5 n-16)-5 \xi^{2}(n-1)(n-4)^{2}\right\} \\
B^{(1, \xi)}\left(0,0 ; p^{2}\right)=0 \\
B^{(1, \xi)}\left(p^{2}, 0 ; 0\right)=-\frac{g^{2} \eta}{(4 \pi)^{n / 2}} C_{A} \frac{1}{64(n-1)(n-4)} \kappa\left\{16\left(4 n^{2}-21 n+14\right)+4 \xi(n-1)\left(10 n^{2}-79 n+152\right)-5 \xi^{2}(n-1)(n-4)^{2}\right\},
\end{gathered}
$$

\footnotetext{
${ }^{18}$ The gauge parameter used in [35] corresponds to our $1-\xi$. To avoid confusion, we shall call their parameter $\xi_{\mathrm{BL}}=1-\xi$. Their constant $T$ corresponds to our $N_{f} T_{R}$. The results presented in [35] are taken at $p^{2}=-\mu^{2}$, where $\mu^{2}$ corresponds to our $\mu_{\mathrm{DR}}^{2}$ (see the discussion in Sec. II). Thus, putting $p^{2}=-\mu^{2}$ formally corresponds to omitting the terms containing $\ln \left(-p^{2}\right)$ [which appear due to the expansion of $\kappa\left(p^{2}\right)$ in $\left.\varepsilon\right]$ in the renormalized expressions.

${ }^{19}$ Their result for $T_{2}$ is proportional to $\left[\left(-\frac{5}{3}+\xi_{\mathrm{BL}}\right) C_{A}+\frac{4}{3} T\right]$, whereas our expressions yield $\left[\left(-\frac{37}{24}+\frac{3}{4} \xi_{\mathrm{BL}}+\frac{1}{8} \xi_{\mathrm{BL}}^{2}\right) C_{A}+\frac{4}{3} T\right]$. So the quark contribution is the same while the sum of gluon and ghost contributions to $T_{2}$ is different. Note that in the Feynman gauge $\left(\xi_{\mathrm{BL}}=1\right)$ our results coincide and yield $\left[-\frac{2}{3} C_{A}+\frac{4}{3} T\right]$. Thus the disagreement does not influence the one-loop part of the two-loop result presented in the Feynman gauge, Eq. (B4) of [35].
} 


$$
\begin{aligned}
& C^{(1, \xi)}\left(0,0 ; p^{2}\right)=\frac{g^{2} \eta}{(4 \pi)^{n / 2}} C_{A} \frac{1}{4(n-4)(n-6) p^{2}} \kappa\left\{12(n-6)+2 \xi\left(n^{2}-11 n+36\right)-\xi^{2}(n-3)(n-8)\right\} \\
& C^{(1, \xi)}\left(p^{2}, 0 ; 0\right)=-\frac{g^{2} \eta}{(4 \pi)^{n / 2}} C_{A} \frac{1}{32(n-1)(n-4) p^{2}} \kappa\left\{16 n(n-4)+4 \xi(n-1)\left(6 n^{2}-41 n+72\right)-3 \xi^{2}(n-1)(n-4)^{2}\right\}, \\
& F^{(1, \xi)}\left(0,0 ; p^{2}\right)=\frac{g^{2} \eta}{(4 \pi)^{n / 2}} C_{A} \frac{1}{2(n-1)(n-4)(n-6)\left(p^{2}\right)^{2}} \kappa\left\{4(n-3)(n-6)(n-13)-2 \xi(n-1)\left(3 n^{2}-20 n+18\right)+\xi^{2}(n-1)\right. \\
& \left.\times\left(n^{3}-16 n^{2}+74 n-78\right)-\xi^{3}(n-1)(n-3)(n-8)\right\}, \\
& F^{(1, \xi)}\left(p^{2}, 0 ; 0\right)=-\frac{g^{2} \eta}{(4 \pi)^{n / 2}} C_{A} \frac{1}{16(n-1)(n-4)(n-6)\left(p^{2}\right)^{2}} \kappa\left\{16(n-6)^{2}(4 n-7)+4 \xi(n-1)(n-6)\left(10 n^{2}-87 n+152\right)\right. \\
& \left.+\xi^{2}(n-1)\left(5 n^{3}-94 n^{2}+504 n-624\right)-8 \xi^{3}(n-1)(n-3)(n-8)\right\}, \\
& H^{(1, \xi)}\left(0,0, p^{2}\right)=-\frac{g^{2} \eta}{(4 \pi)^{n / 2}} C_{A} \frac{1}{16(n-1)(n-4)(n-6) p^{2}} \kappa\left\{16(n-4)(n-6)(n+5)+24 \xi(n-1)(n-6)\left(n^{2}-6 n+12\right)\right. \\
& \left.-\xi^{2}(n-1)\left(7 n^{3}-110 n^{2}+532 n-696\right)+4 \xi^{3}(n-1)(n-3)(n-8)\right\} .
\end{aligned}
$$

The quark loop contributions yield

$$
\begin{gathered}
A^{(1, q)}\left(0,0 ; p^{2}\right)=B^{(1, q)}\left(0,0 ; p^{2}\right)=C^{(1, q)}\left(0,0 ; p^{2}\right)=0 \\
A^{(1, q)}\left(p^{2}, 0 ; 0\right)=B^{(1, q)}\left(p^{2}, 0 ; 0\right)=\frac{g^{2} \eta}{(4 \pi)^{n / 2}} N_{f} T_{R} \frac{n-2}{n-1} \kappa \\
C^{(1, q)}\left(p^{2}, 0 ; 0\right)=\frac{g^{2} \eta}{(4 \pi)^{n / 2}} 2 N_{f} T_{R} \frac{n-2}{n-1} \frac{\kappa}{p^{2}}, \\
F^{(1, q)}\left(0,0 ; p^{2}\right)=\frac{g^{2} \eta}{(4 \pi)^{n / 2}} 64 N_{f} T_{R} \frac{1}{(n-1)(n-2)} \frac{\kappa}{\left(p^{2}\right)^{2}}, \\
F^{(1, q)}\left(p^{2}, 0 ; 0\right)=\frac{g^{2} \eta}{(4 \pi)^{n / 2}} 4 N_{f} T_{R} \frac{n(n-6)}{(n-1)(n-2)} \frac{\kappa}{\left(p^{2}\right)^{2}}, \\
H^{(1, q)}\left(0,0, p^{2}\right)=\frac{g^{2} \eta}{(4 \pi)^{n / 2}} 4 N_{f} T_{R} \frac{n^{2}-3 n+8}{(n-1)(n-2) p^{2}}
\end{gathered}
$$

Since in the limit $p_{1}^{2}=p_{2}^{2}=0$ the scalar functions may depend on $p_{3}^{2} \equiv p^{2}$ only, the independent tensor structures (expressed in terms of $p_{1}$ and $\left.p_{2}\right)$ can be chosen antisymmetric with respect to the permutation $\left(p_{1}, \mu_{1}\right) \leftrightarrow\left(p_{2}, \mu_{2}\right)$. The three-gluon vertex can in this limit be written as

$$
\begin{aligned}
\left.\Gamma_{\mu_{1} \mu_{2} \mu_{3}}\left(p_{1}, p_{2}, p_{3}\right)\right|_{p_{1}^{2}=p_{2}^{2}=0} & g_{\mu_{1} \mu_{2}}\left(p_{1}-p_{2}\right)_{\mu_{3}} U_{1}\left(p^{2}\right)+\left[g_{\mu_{1} \mu_{3}} p_{1 \mu_{2}}-g_{\mu_{2} \mu_{3}} p_{2 \mu_{1}}\right] U_{2}\left(p^{2}\right) \\
& +\left[g_{\mu_{1} \mu_{3}} p_{2 \mu_{2}}-g_{\mu_{2} \mu_{3}} p_{1 \mu_{1}}\right] U_{3}\left(p^{2}\right)+p_{1 \mu_{1}} p_{2 \mu_{2}}\left(p_{1}-p_{2}\right)_{\mu_{3}} U_{4}\left(p^{2}\right) \\
& +p_{1 \mu_{2}} p_{2 \mu_{1}}\left(p_{1}-p_{2}\right)_{\mu_{3}} U_{5}\left(p^{2}\right)+\left[p_{1 \mu_{1}} p_{1 \mu_{2}} p_{1 \mu_{3}}-p_{2 \mu_{1}} p_{2 \mu_{2}} p_{2 \mu_{3}}\right] U_{6}\left(p^{2}\right) \\
& +\left[p_{1 \mu_{1}} p_{1 \mu_{2}} p_{2 \mu_{3}}-p_{2 \mu_{1}} p_{2 \mu_{2}} p_{1 \mu_{3}}\right] U_{7}\left(p^{2}\right)
\end{aligned}
$$


This decomposition is analogous to Eq. (29) of [14], and the functions $U_{i}$ are proportional to the functions $F_{j}$ used in [14] (we have different numbering; see in Appendix F).

The following representations of the $U_{i}$ functions in terms of scalar functions corresponding to the decomposition (2.4) can be derived in this limit:

$$
\begin{gathered}
U_{1}\left(p^{2}\right)=A\left(0,0 ; p^{2}\right)-\frac{1}{2} p^{2} C\left(0,0 ; p^{2}\right)-\frac{1}{4}\left(p^{2}\right)^{2} F\left(0,0 ; p^{2}\right) \\
+ \\
\quad \frac{1}{2} p^{2} H\left(0,0, p^{2}\right), \\
U_{2}\left(p^{2}\right)=-2 A\left(p^{2}, 0 ; 0\right)-p^{2} C\left(p^{2}, 0 ; 0\right), \\
U_{3}\left(p^{2}\right)=-A\left(p^{2}, 0 ; 0\right)-B\left(p^{2}, 0 ; 0\right)-\frac{1}{2} p^{2} C\left(p^{2}, 0 ; 0\right) \\
\quad+\frac{1}{4}\left(p^{2}\right)^{2} F\left(p^{2}, 0 ; 0\right)+\frac{1}{2} p^{2} H\left(0,0, p^{2}\right), \\
U_{4}\left(p^{2}\right)=C\left(p^{2}, 0 ; 0\right)-\frac{1}{2} p^{2} F\left(p^{2}, 0 ; 0\right), \\
U_{5}\left(p^{2}\right)=2 C\left(p^{2}, 0 ; 0\right)+C\left(0,0 ; p^{2}\right)+\frac{1}{2} p^{2} F\left(0,0 ; p^{2}\right) \\
\quad-H\left(0,0, p^{2}\right), \\
U_{6}\left(p^{2}\right)=2 C\left(p^{2}, 0 ; 0\right), \\
U_{7}\left(p^{2}\right)=-C\left(p^{2}, 0 ; 0\right)+\frac{1}{2} p^{2} F\left(p^{2}, 0 ; 0\right)+H\left(0,0, p^{2}\right) .
\end{gathered}
$$

The explicit results for the $U_{i}$ functions are presented in Appendix F. The infrared-divergent contributions were successfully compared with Eq. (30) of [14], where the corresponding contributions to $F_{j}$ are presented. Note that $U_{4}, U_{5}, U_{6}$, and $U_{7}$ can be directly compared with [14], while the expressions for $U_{1}, U_{2}$, and $U_{3}$ (containing the $A$ function) should be renormalized by adding the conterterm contribution (3.38) to all $A$ functions involved.

The result for the three-gluon vertex in the Feynman gauge (for $p_{1}^{2}=p_{2}^{2}=0$ ) is available in Appendix B of Ref. [15]. It is expanded around $n=4$, and the divergent and finite (in $\varepsilon$ ) parts are presented. In this limit, our expressions yield the same as the results of [15].

\section{CONCLUSIONS}

In this paper, we have presented results for the one-loop three-gluon vertex valid for arbitrary values of the spacetime dimension, $n$, and the covariant-gauge parameter, $\xi$. We have considered the general off-shell case (arbitrary $p_{1}^{2}, p_{2}^{2}$, and $p_{3}^{2}$; see Sec. III), as well as all on-shell cases of interest (Sec. IV). Moreover, having the results in arbitrary dimension, it was possible to get all on-shell expressions just by considering the corresponding limits of the general (offshell) results. This would be impossible if one started from the off-shell results expanded around $n=4$, because in this case the infrared (on-shell) divergences would appear as logarithms of vanishing momenta squared. The only restriction we used in our calculations was that in the quark loop contributions the quarks were taken to be massless. ${ }^{20}$

To calculate the vertex, we used the decomposition (2.4) (adopted from Ref. [13]) and considered the six scalar functions $A, B, C, S, F$, and $H$, which completely define the three-gluon vertex. One of these functions, namely, the $S$ function, was found to be identically zero at the one-loop order, ${ }^{21}$ see Eqs. (3.13) and (3.20). We have also checked that $S=0$ when massive quarks are considered. It is not clear whether it vanishes also at the two-loop level. For the five remaining functions $A, B, C, F$, and $H$, the general offshell results are given in Eqs. (3.4)-(3.9) (Feynman gauge), Eqs. (3.10)-(3.15) (arbitrary gauge), and Eqs. (3.17)-(3.22) (quark loop contributions). They involve only one nontrivial function $\varphi\left(p_{1}^{2}, p_{2}^{2}, p_{3}^{2}\right)$ [see Eq. (2.14) and Appendix B], which is related to the scalar one-loop triangle diagram. For special cases, we have successfully compared our results with those from Refs. [11,13] (for details, see Sec. III).

Starting from general expressions and putting some external momenta squared equal to zero, we considered the onshell cases $p_{3}^{2}=0$ (Sec. IV A), $p_{3}=0$ (Sec. IV B), and $p_{1}^{2}=p_{2}^{2}=0$ (Sec. IV C). For all these cases, the results in arbitrary gauge and dimension were presented; see Sec. IV and Appendixes E and F. For special cases, our results have been compared with those presented in Refs. [14,15]. Thus, we can see that Table I from the Introduction is completely filled in. Moreover, all results are valid for an arbitrary value of the space-time dimension. Thus, the only thing which at the one-loop level is left for the future is to allow for nonzero quark masses in the quark loops.

Furthermore, we have obtained general results for the ghost-gluon vertex (2.10); see Eqs. (D7)-(D11) in Appendix D. Employing these results, together with two-point contributions listed in Appendix $\mathrm{C}$, we have checked that the Ward-Slavnov-Taylor identity (2.12) for the three-gluon vertex is exactly (i.e., for arbitrary $n$ and $\xi$ ) satisfied by the expressions obtained, as it has to. This was another nontrivial check on the longitudinal part of the vertex $(A, B, C$ and $S$ functions).

We note that techniques have recently become available $[36,37]$ to study the off-shell massless vertices at the twoloop level, at least in the Feynman gauge. Here, the main difficulty is integrals with higher powers of irreducible numerators [37].

In the future, the one-loop quark-gluon vertex can also be considered in a similar way. For the case of massless quarks, one can use the same approach as in this paper. For massive quarks, one should study in more detail what the corresponding scalar integrals in arbitrary dimension are. ${ }^{22}$ We note that

\footnotetext{
${ }^{20}$ This restriction does not affect any of the results for the gluon and ghost loop contributions [the functions marked $(1, \xi)]$ which are indeed the most general ones.

${ }^{21}$ In the Feynman gauge and four dimensions, this result was obtained in [13].

${ }^{22}$ This is also the reason why we did not consider massive quark loops in this paper.
} 
some results for the quark-gluon vertex (and also for the QED vertex which formally corresponds to one of the two diagrams contributing to the quark-gluon vertex) can be found, e.g., in $[38,35]$.

\section{ACKNOWLEDGMENTS}

The authors are indebted to J.S. Ball and T.-W. Chiu for useful communications and, in particular, for confirming two minor misprints in [13] mentioned in Sec. III A and Appendix D. O.T. is grateful to NORDITA for supporting his visit to Bergen where this work was initiated, and to the University of Bergen for hospitality. A.D.'s and P.O.'s research was supported by the Research Council of Norway.

\section{APPENDIX A: \\ DECOMPOSITION OF THREE-GLUON VERTEX}

If we express $p_{3}$ in terms of the two other momenta, $p_{3}=-p_{1}-p_{2}$, we get the following decompostion of the three-gluon vertex (2.2):

$$
\begin{aligned}
\Gamma_{\mu_{1} \mu_{2} \mu_{3}}\left(p_{1}, p_{2}, p_{3}\right)= & g_{\mu_{1} \mu_{2}} p_{1 \mu_{3}} Z_{001}+g_{\mu_{1} \mu_{3}} p_{1 \mu_{2}} Z_{010}+g_{\mu_{2} \mu_{3}} p_{1 \mu_{1}} Z_{100}+g_{\mu_{1} \mu_{2}} p_{2 \mu_{3}} Z_{002}+g_{\mu_{1} \mu_{3}} p_{2 \mu_{2}} Z_{020}+g_{\mu_{2} \mu_{3}} p_{2 \mu_{1}} Z_{200} \\
& +p_{1 \mu_{1}} p_{1 \mu_{2}} p_{1 \mu_{3}} Z_{111}+p_{2 \mu_{1}} p_{2 \mu_{2}} p_{2 \mu_{3}} Z_{222}+p_{1 \mu_{1}} p_{1 \mu_{2}} p_{2 \mu_{3}} Z_{112}+p_{1 \mu_{1}} p_{2 \mu_{2}} p_{1 \mu_{3}} Z_{121} \\
& +p_{2 \mu_{1}} p_{1 \mu_{2}} p_{1 \mu_{3}} Z_{211}+p_{1 \mu_{1}} p_{2 \mu_{2}} p_{2 \mu_{3}} Z_{122}+p_{2 \mu_{1}} p_{1 \mu_{2}} p_{2 \mu_{3}} Z_{212}+p_{2 \mu_{1}} p_{2 \mu_{2}} p_{1 \mu_{3}} Z_{221}
\end{aligned}
$$

where $Z_{j k l}$ are scalar functions depending on $p_{1}^{2}, p_{2}^{2}$, and $p_{3}^{2}$.

Comparison with the decomposition (2.4) used in [13] gives the following representations of $Z$ 's in terms of the functions (2.4) used by Ball and Chiu [13]:

$$
\left.\begin{array}{rl}
Z_{001}= & A\left(p_{1}^{2}, p_{2}^{2} ; p_{3}^{2}\right)-\left(p_{1} p_{2}\right) C\left(p_{1}^{2}, p_{2}^{2} ; p_{3}^{2}\right)+B\left(p_{1}^{2}, p_{2}^{2} ; p_{3}^{2}\right) \\
& +\left(p_{1} p_{2}\right)\left(p_{2} p_{3}\right) F\left(p_{1}^{2}, p_{2}^{2} ; p_{3}^{2}\right)-\left(p_{2} p_{3}\right) H, \\
Z_{002}= & -A\left(p_{1}^{2}, p_{2}^{2} ; p_{3}^{2}\right)+\left(p_{1} p_{2}\right) C\left(p_{1}^{2}, p_{2}^{2} ; p_{3}^{2}\right)+B\left(p_{1}^{2}, p_{2}^{2} ; p_{3}^{2}\right) \\
& -\left(p_{1} p_{2}\right)\left(p_{1} p_{3}\right) F\left(p_{1}^{2}, p_{2}^{2} ; p_{3}^{2}\right)+\left(p_{1} p_{3}\right) H, \\
Z_{100}= & A\left(p_{2}^{2}, p_{3}^{2} ; p_{1}^{2}\right)-\left(p_{2} p_{3}\right) C\left(p_{2}^{2}, p_{3}^{2} ; p_{1}^{2}\right)-B\left(p_{2}^{2}, p_{3}^{2} ; p_{1}^{2}\right) \\
& +\left(p_{1} p_{2}\right)\left(p_{2} p_{3}\right) F\left(p_{2}^{2}, p_{3}^{2} ; p_{1}^{2}\right)-\left(p_{1} p_{2}\right) H, \\
& Z_{200}=2 A\left(p_{2}^{2}, p_{3}^{2} ; p_{1}^{2}\right)-2\left(p_{2} p_{3}\right) C\left(p_{2}^{2}, p_{3}^{2} ; p_{1}^{2}\right) \\
& -p_{1}^{2}\left(p_{2} p_{3}\right) F\left(p_{2}^{2}, p_{3}^{2} ; p_{1}^{2}\right)+p_{1}^{2} H, \\
Z_{010}= & -2 A\left(p_{3}^{2}, p_{1}^{2} ; p_{2}^{2}\right)+2\left(p_{1} p_{3}\right) C\left(p_{3}^{2}, p_{1}^{2} ; p_{2}^{2}\right) \\
& +p_{2}^{2}\left(p_{1} p_{3}\right) F\left(p_{3}^{2}, p_{1}^{2} ; p_{2}^{2}\right)-p_{2}^{2} H, \\
Z_{020}=- & A\left(p_{3}^{2}, p_{1}^{2} ; p_{2}^{2}\right)+\left(p_{1} p_{3}\right) C\left(p_{3}^{2}, p_{1}^{2} ; p_{2}^{2}\right)-B\left(p_{3}^{2}, p_{1}^{2} ; p_{2}^{2}\right) \\
- & \left(p_{1} p_{2}\right)\left(p_{1} p_{3}\right) F\left(p_{3}^{2}, p_{1}^{2} ; p_{2}^{2}\right)+\left(p_{1} p_{2}\right) H, \\
& Z_{111}=2 C\left(p_{3}^{2}, p_{1}^{2} ; p_{2}^{2}\right)+p_{2}^{2} F\left(p_{3}^{2}, p_{1}^{2} ; p_{2}^{2}\right), \\
& Z_{222}=-2 C\left(p_{2}^{2}, p_{3}^{2} ; p_{1}^{2}\right)-p_{1}^{2} F\left(p_{2}^{2}, p_{3}^{2} ; p_{1}^{2}\right), \\
Z_{112}= & -C\left(p_{2}^{2}, p_{3}^{2} ; p_{1}^{2}\right)+\left(p_{1} p_{2}\right) F\left(p_{2}^{2}, p_{3}^{2} ; p_{1}^{2}\right)+H-S, \\
Z_{121}= & C\left(p_{3}^{2}, p_{1}^{2} ; p_{2}^{2}\right)-\left(p_{1} p_{2}\right) F\left(p_{3}^{2}, p_{1}^{2} ; p_{2}^{2}\right), \quad(\mathrm{A} 10) \\
& -C\left(p_{2}^{2}, p_{3}^{2} ; p_{1}^{2}\right)+\left(p_{1} p_{2}\right) F\left(p_{2}^{2}, p_{3}^{2} ; p_{1}^{2}\right), \quad(\mathrm{A} 12)
\end{array}\right)
$$

$$
\begin{aligned}
Z_{211}= & C\left(p_{1}^{2}, p_{2}^{2} ; p_{3}^{2}\right)+2 C\left(p_{3}^{2}, p_{1}^{2} ; p_{2}^{2}\right)-\left(p_{2} p_{3}\right) F\left(p_{1}^{2}, p_{2}^{2} ; p_{3}^{2}\right) \\
+ & p_{2}^{2} F\left(p_{3}^{2}, p_{1}^{2} ; p_{2}^{2}\right)-H-S, \\
Z_{212}= & -C\left(p_{1}^{2}, p_{2}^{2} ; p_{3}^{2}\right)-2 C\left(p_{2}^{2}, p_{3}^{2} ; p_{1}^{2}\right) \\
& +\left(p_{1} p_{3}\right) F\left(p_{1}^{2}, p_{2}^{2} ; p_{3}^{2}\right)-p_{1}^{2} F\left(p_{2}^{2}, p_{3}^{2} ; p_{1}^{2}\right)+H-S \\
Z_{221} & =C\left(p_{3}^{2}, p_{1}^{2} ; p_{2}^{2}\right)-\left(p_{1} p_{2}\right) F\left(p_{3}^{2}, p_{1}^{2} ; p_{2}^{2}\right)-H-S,
\end{aligned}
$$

where $H \equiv H\left(p_{1}^{2}, p_{2}^{2}, p_{3}^{2}\right)$ and $S \equiv S\left(p_{1}^{2}, p_{2}^{2}, p_{3}^{2}\right)$.

Solving these equations we get the following results for the scalar functions (2.4), including those with permuted arguments, in terms of $Z$ 's:

$$
\begin{gathered}
S\left(p_{1}^{2}, p_{2}^{2}, p_{3}^{2}\right)=\frac{1}{2}\left\{-Z_{112}+Z_{121}+Z_{122}-Z_{221}\right\}, \\
H\left(p_{1}^{2}, p_{2}^{2}, p_{3}^{2}\right)=\frac{1}{2}\left\{Z_{112}+Z_{121}-Z_{122}-Z_{221}\right\}, \\
A\left(p_{1}^{2}, p_{2}^{2} ; p_{3}^{2}\right)=\frac{1}{2}\left\{\left(p_{1} p_{2}\right)\left[-Z_{111}+Z_{222}+Z_{211}-Z_{212}\right]\right. \\
\left.+Z_{001}-Z_{002}-\left(p_{1}^{2}+p_{2}^{2}\right) H\right\}, \\
A\left(p_{2}^{2}, p_{3}^{2} ; p_{1}^{2}\right)=\frac{1}{2}\left\{-\left(p_{2} p_{3}\right) Z_{222}+Z_{200}-p_{1}^{2} H\right\}, \\
A\left(p_{3}^{2}, p_{1}^{2} ; p_{2}^{2}\right)=\frac{1}{2}\left\{\left(p_{1} p_{3}\right) Z_{111}+Z_{010}-p_{2}^{2} H\right\}, \\
B\left(p_{1}^{2}, p_{2}^{2} ; p_{3}^{2}\right)=\frac{1}{2}\left\{p_{1}^{2}\left[Z_{112}-Z_{122}\right]+p_{2}^{2}\left[-Z_{121}+Z_{221}\right]\right. \\
-\left(p_{1} p_{2}\right)\left[Z_{111}+Z_{222}-Z_{211}-Z_{212}\right]+Z_{001} \\
\left.+Z_{002}+p_{3}^{2} S\right\},
\end{gathered}
$$




$$
\begin{aligned}
& B\left(p_{2}^{2}, p_{3}^{2} ; p_{1}^{2}\right)=\frac{1}{2}\left\{-\left(p_{2} p_{3}\right)\left(Z_{222}-2 Z_{122}\right)+Z_{200}-2 Z_{100}\right. \\
& \left.+\left(p_{2}^{2}-p_{3}^{2}\right) H\right\}, \\
& B\left(p_{3}^{2}, p_{1}^{2} ; p_{2}^{2}\right)=\frac{1}{2}\left\{-\left(p_{1} p_{3}\right)\left(Z_{111}-2 Z_{121}\right)+Z_{010}-2 Z_{020}\right. \\
& \left.+\left(p_{3}^{2}-p_{1}^{2}\right) H\right\}, \\
& C\left(p_{1}^{2}, p_{2}^{2} ; p_{3}^{2}\right)=\frac{1}{p_{1}^{2}-p_{2}^{2}}\left\{\left(p_{1} p_{3}\right)\left[Z_{111}-Z_{211}-Z_{121}+Z_{221}\right]\right. \\
& \left.+\left(p_{2} p_{3}\right)\left[Z_{222}+Z_{112}-Z_{122}-Z_{212}\right]\right\}, \\
& C\left(p_{2}^{2}, p_{3}^{2} ; p_{1}^{2}\right)=\frac{1}{p_{2}^{2}-p_{3}^{2}}\left\{\left(p_{1} p_{2}\right) Z_{222}+p_{1}^{2} Z_{122}\right\}, \\
& C\left(p_{3}^{2}, p_{1}^{2} ; p_{2}^{2}\right)=\frac{1}{p_{3}^{2}-p_{1}^{2}}\left\{\left(p_{1} p_{2}\right) Z_{111}+p_{2}^{2} Z_{121}\right\}, \\
& F\left(p_{1}^{2}, p_{2}^{2} ; p_{3}^{2}\right)=\frac{1}{p_{1}^{2}-p_{2}^{2}}\left\{Z_{111}+Z_{222}+Z_{112}-Z_{121}-Z_{211}-Z_{122}\right. \\
& \left.-Z_{212}+Z_{221}\right\} \text {, } \\
& F\left(p_{2}^{2}, p_{3}^{2} ; p_{1}^{2}\right)=\frac{1}{p_{2}^{2}-p_{3}^{2}}\left\{Z_{222}-2 Z_{122}\right\}, \\
& F\left(p_{3}^{2}, p_{1}^{2} ; p_{2}^{2}\right)=\frac{1}{p_{3}^{2}-p_{1}^{2}}\left\{Z_{111}-2 Z_{121}\right\} .
\end{aligned}
$$

\section{APPENDIX B: SCALAR INTEGRALS}

As mentioned in Sec. II, the results for the scalar functions occurring in Eqs. (2.4) and (2.10) can be represented in terms of the following Feynman integrals, corresponding to a scalar one-loop triangle diagram:

$$
J\left(\nu_{1}, \nu_{2}, \nu_{3}\right) \equiv \int \frac{d^{n} q}{\left[\left(p_{2}-q\right)^{2}\right]^{\nu_{1}}\left[\left(p_{1}+q\right)^{2}\right]^{\nu_{2}}\left(q^{2}\right)^{\nu_{3}}},
$$

where $n=4-2 \varepsilon$ is the space-time dimension.

When we perform calculations in the Feynman gauge and express the scalar numerators in terms of the denominators, the powers of the denominators, $\nu_{i}$, can be 1,0 , or even negative. In an arbitrary gauge, the integrals may have powers of $\nu_{i}$ equal to 2, due to the presence of $\left(p^{2}\right)^{-2}$ in the $\xi$ term of the gluon propagator, Eq. (2.3). Nevertheless, by using the integration-by-parts technique [27] these integrals can be reduced to those with $\nu$ 's equal to 1 or 0 . The corresponding algorithm for the integrals (B1) is described in detail in $[28] .^{23}$

Then, if two or three $\nu$ 's are nonpositive integers, the dimensionally regularized integral (B1) vanishes [23], since it corresponds to a massless tadpole. When one of the $\nu$ 's is

\footnotetext{
${ }^{23}$ The main formula to be used is Eq. (3.4) of [28]. Some explicit results for the integrals with $\nu_{i}=2$ are also presented in [28].
}

0 , the integral (B1) corresponds to a two-point function. Therefore, it is proportional to a power of the external momentum squared times some $\Gamma$ functions with arguments involving $n$ and $\nu$ 's. We mainly need the result for two remaining $\nu$ 's equal to 1 [e.g., $J(1,1,0)]$ which is given by Eqs. (2.15) and (2.16), in which case this $\Gamma$ factor is nothing but $\eta$, Eq. (2.17).

Then, the integrals with one negative $\nu$ can easily be reduced to integrals with the corresponding $\nu$ equal to 0 , for example,

$$
J(1,1,-1)=-\left(p_{1} p_{2}\right) J(1,1,0),
$$

$$
\begin{aligned}
J(1,1,-2)= & \frac{1}{n-1}\left[n\left(p_{1} p_{2}\right)^{2}-p_{1}^{2} p_{2}^{2}\right] J(1,1,0), \\
J(1,1,-3)= & -\frac{1}{n-1}\left[(n+2)\left(p_{1} p_{2}\right)^{2}-3 p_{1}^{2} p_{2}^{2}\right] \\
& \times\left(p_{1} p_{2}\right) J(1,1,0) .
\end{aligned}
$$

Thus, the only nontrivial function which occurs in our calculations is $\varphi$ which is related to the triangle integral $J(1,1,1)[$ cf. (2.14)] via

$$
J(1,1,1)=i \pi^{n / 2} \eta \varphi\left(p_{1}^{2}, p_{2}^{2}, p_{3}^{2}\right),
$$

where $\eta$ is defined by Eq. (2.17).

In fact, the general results (i.e., for arbitrary $n, \nu_{i}$, and $p_{i}^{2}$ ) for the integrals (B1) are available [39]. They can be represented in terms of Appell's hypergeometric function of two variables, $F_{4}$. As dimensionless variables, one can use

$$
x \equiv \frac{p_{1}^{2}}{p_{3}^{2}} \text { and } y \equiv \frac{p_{2}^{2}}{p_{3}^{2}} .
$$

When $\nu_{1}=\nu_{2}=\nu_{3}=1$, all the $F_{4}$ functions can be reduced to ${ }_{2} F_{1}$ Gauss hypergeometric functions of more complicated arguments, by using reduction formulas for $F_{4}$ functions (see, e.g., Ref. [40]). One can also derive a one-dimensional integral representation [see Eq. (26) of [37]] which is valid for arbitrary $\varepsilon=(4-n) / 2$ and, therefore, for arbitrary $n$,

$$
J(1,1,1)=-\frac{i \pi^{2-\varepsilon} \eta}{\left(-p_{3}^{2}\right)^{1+\varepsilon}} \frac{1}{\varepsilon} \int_{0}^{1} \frac{d \sigma \sigma^{-\varepsilon}\left[(y \sigma)^{-\varepsilon}-(x / \sigma)^{-\varepsilon}\right]}{\left[y \sigma^{2}+(1-x-y) \sigma+x\right]^{1-\varepsilon}},
$$

with $x$ and $y$ defined by Eq. (B6).

Another way to get the result for arbitrary $n$ is to use the connection [41] between massless triangle integrals and the two-loop massive vacuum integrals, $I\left(\nu_{1}, \nu_{2}, \nu_{3}\right)$. In particular, according to Eq. (40) of [41], for arbitrary $n$ the integral $J(1,1,1)$ can be related (up to trivial leftovers) to the integral $I(1,1,1)$, in such a way that one of the integrals is taken in $4-2 \varepsilon$ dimensions, and the other in $4+2 \varepsilon$ dimensions. Using this connection, and also known results for $I(1,1,1)$ in arbitrary dimension [42-44], one can reproduce the result for $J(1,1,1)$. It can be written in terms of ${ }_{2} F_{1}$ functions (see, e.g., Eq. (4.12) of [44]).

The result for $J(1,1,1)$ in four dimensions is well known $[45,13]$ (see also Refs. $[28,36]$ ) and can be presented in terms of dilogarithms, 


$$
\begin{gathered}
\left.\varphi\left(p_{1}^{2}, p_{2}^{2}, p_{3}^{2}\right)\right|_{n=4}=\frac{1}{p_{3}^{2} \lambda}\left\{2\left[\operatorname{Li}_{2}(-\rho x)+\mathrm{Li}_{2}(-\rho y)\right]\right. \\
\left.+\ln \frac{y}{x} \ln \frac{1+\rho y}{1+\rho x}+\ln (\rho x) \ln (\rho y)+\frac{\pi^{2}}{3}\right\},
\end{gathered}
$$

where

$$
\lambda(x, y) \equiv \sqrt{(1-x-y)^{2}-4 x y}, \quad \rho(x, y) \equiv \frac{2}{1-x-y+\lambda},
$$

or, in terms of the Clausen function $\mathrm{Cl}_{2}$ [see, e.g., Eq. (19) of [41]; similar representations are also given in $[42,46]]$. In particular, in the symmetric case (see Sec. III D)

$$
\begin{aligned}
\left.J(1,1,1)\right|_{\substack{p_{1}^{2}=p_{2}^{2}=p_{3}^{2} \equiv p^{2} \\
n=4}} & \left.i \pi^{2} \varphi\left(p^{2}, p^{2}, p^{2}\right)\right|_{n=4} \\
& =\frac{i \pi^{2}}{p^{2}} \frac{4}{\sqrt{3}} \mathrm{Cl}_{2}\left(\frac{\pi}{3}\right),
\end{aligned}
$$

producing the same constant as the one (denoted as $I$ ) used in [11]. The transcendental number $\mathrm{Cl}_{2}(\pi / 3)$ $=1.0149417 \ldots$ corresponds to the maximum of Clausen's integral and appears frequently in two-loop calculations with masses (see, e.g., [47,42]), the connection with the massless triangle diagrams being clear from [41].

If one is interested in expanding in $\varepsilon$ and calculating the integral $J(1,1,1)$ up to order $\varepsilon$, one can use Eq. (29) of [37] or Eqs. (16) and (20) of [41].

In three dimensions, the result for the integral $J(1,1,1)$ is very simple [48] and proportional to $\left(p_{1}^{2} p_{2}^{2} p_{3}^{2}\right)^{-1 / 2}$. To get the result around two dimensions, Eq. (43) of [41] can be used. $^{24}$ The corresponding two-dimensional integral has infrared singularities which can be regularized by the same $\varepsilon$. However, the only nontrivial function is the same as in the four-dimensional case. The same equation (43) of [41] can also be used to get the results for higher values of $n$.

When some of the external momenta squared vanish, the integral $J(1,1,1)$ (considered in four dimensions) develops infrared (on-shell) singularities. The corresponding limits are considered in Sec. IV.

\section{APPENDIX C: TWO-POINT FUNCTIONS}

To check whether our results are consistent with the Ward-Slavnov-Taylor identity for the three-gluon vertex (2.12), we need expressions for the functions contributing to the gluon polarization operator and the ghost self-energy.

The corresponding scalar functions $J\left(p^{2}\right)$ and $G\left(p^{2}\right)$ are defined in Eqs. (2.7) and (2.8), respectively. The lowestorder results are $J^{(0)}=G^{(0)}=1$. At the one-loop order, the results can be found, e.g., in [8]. We present them here for completeness and also to show the proper normalization of the functions. One-loop contributions to these functions are presented in Fig. 2.

\footnotetext{
${ }^{24}$ This is a special case of a more general result presented in [49].
}

The gluon and ghost loop contributions to the $J$ and $G$ function in an arbitrary covariant gauge yield

$$
\begin{gathered}
J^{(1, \xi)}\left(p^{2}\right)=-\frac{g^{2} \eta}{(4 \pi)^{n / 2}} \frac{C_{A}}{8(n-1)}\{4(3 n-2)+4 \xi(n-1)(2 n \\
\left.-7)-\xi^{2}(n-1)(n-4)\right\} \kappa\left(p^{2}\right), \\
G^{(1, \xi)}\left(p^{2}\right)=\frac{g^{2} \eta}{(4 \pi)^{n / 2}} \frac{C_{A}}{4}[2+\xi(n-3)] \kappa\left(p^{2}\right), \quad(\mathrm{C} 2)
\end{gathered}
$$

while the quark loop contribution to the $J$ function is

$$
J^{(1, q)}\left(p^{2}\right)=\frac{g^{2} \eta}{(4 \pi)^{n / 2}} 2 N_{f} T_{R} \frac{n-2}{n-1} \kappa\left(p^{2}\right)
$$

(there is no quark contribution to the $G$ function). The coefficients $\eta, C_{A}$, and $T_{R}$ are defined by Eqs. (2.17), (3.3), and (3.16), respectively. The function $\kappa\left(p^{2}\right)$ is given by Eq. (2.15). by

The ultraviolet divergences of Eqs. (C1)-(C3) are given

$$
\begin{gathered}
J^{(1, \mathrm{UV})}=\frac{g^{2} \eta}{(4 \pi)^{2-\varepsilon}}\left[-C_{A}\left(\frac{5}{3}+\frac{1}{2} \xi\right)+\frac{4}{3} N_{f} T_{R}\right] \kappa^{(\mathrm{UV})}, \\
G^{(1, \mathrm{UV})}=\frac{g^{2} \eta}{(4 \pi)^{2-\varepsilon}} C_{A} \frac{1}{4}(2+\xi) \kappa^{(\mathrm{UV})}
\end{gathered}
$$

where $\kappa^{(\mathrm{UV})}=1 / \varepsilon+\cdots$ is the divergent part of $\kappa\left(p^{2}\right)$, Eq. (2.15). The corresponding counterterms are

$$
\begin{aligned}
J^{(1, \mathrm{CT})} & =\frac{\bar{g}^{2}}{(4 \pi)^{2}}\left[C_{A}\left(\frac{5}{3}+\frac{1}{2} \xi\right)-\frac{4}{3} N_{f} T_{R}\right]\left(\frac{1}{\varepsilon}+R\right) \\
& =\frac{g^{2} \eta}{(4 \pi)^{2-\varepsilon}}\left[C_{A}\left(\frac{5}{3}+\frac{1}{2} \xi\right)-\frac{4}{3} N_{f} T_{R}\right]\left(\frac{1}{\varepsilon}+R\right)+O(\varepsilon), \\
G^{(1, \mathrm{CT})} & =-\frac{\bar{g}^{2}}{(4 \pi)^{2}} \frac{C_{A}}{4}(2+\xi)\left(\frac{1}{\varepsilon}+R\right) \\
& =-\frac{g^{2} \eta}{(4 \pi)^{2-\varepsilon}} \frac{C_{A}}{4}(2+\xi)\left(\frac{1}{\varepsilon}+R\right)+O(\varepsilon),(\mathrm{C} 5)
\end{aligned}
$$

where $\bar{g}^{2} \equiv g^{2} e^{-\gamma \varepsilon}(4 \pi)^{\varepsilon}$ and $R$ is the renormalizationscheme constant chosen in such a way that $R=0$ in the $\overline{\mathrm{MS}}$ scheme (see also Sec. III E).

Note that in the Fried-Yennie gauge ${ }^{25}[31], \xi=-2$, the ghost self-energy is finite as $n \rightarrow 4$. Moreover, if one chooses the $n$-dimensional generalization of this gauge as $\xi=-2 /(n-3)$ [50], then the one-loop correction to the ghost self-energy vanishes. This is connected with the transversality of the gluon propagator (2.3) in the coordinate space (at this value of $\xi$ ).

The gluon polarization operator is finite when

$$
\left.\xi\right|_{n=4} \equiv \xi_{0}=\frac{2}{3}\left(\frac{4 N_{f} T_{R}}{C_{A}}-5\right) .
$$

\footnotetext{
${ }^{25}$ This gauge was also used in Ref. [32].
} 
For $N_{f}=6, T_{R}=\frac{1}{2}$, and $C_{A}=3$, this value is $\xi_{0}=-\frac{2}{3}$.

\section{APPENDIX D: \\ RESULTS FOR THE GHOST-GLUON VERTEX}

There are two one-loop contributions to the ghost-gluon vertex which are shown in Fig. 3. Here, we present the most general results for the scalar functions contributing to the ghost-gluon vertex at the one-loop level. The definition of the ghost-gluon vertex and the decomposition in terms of scalar functions is given in Eqs. (2.9) and (2.10), respectively. The lowest-order expression is given by Eq. (2.11). In one-loop expressions, we use the notation for $\varphi, \kappa_{i}$, and $\eta$ which can be found in Eqs. (2.14), (2.15), and (2.17), respectively. $\mathcal{K}$ and $\mathcal{Q}$ denote the symmetric scalar combinations constructed from the external momenta, Eqs. (3.2) and (3.1).

At the one-loop level (in the Feynman gauge) we get

$$
\begin{aligned}
\widetilde{\Gamma}_{\mu \mu_{3}}^{(1,0)}\left(p_{1}, p_{2} ; p_{3}\right)= & -\frac{g^{2} \eta}{(4 \pi)^{n / 2}} C_{A} \frac{1}{4 \mathcal{K}}\left(g_{\mu \mu_{3}} \mathcal{K}\left[\left(2 p_{1}^{2}-p_{2}^{2}\right) \varphi+\kappa_{1}-2 \kappa_{2}-\kappa_{3}\right]+p_{1 \mu} p_{1 \mu_{3}}\left[p_{2}^{2}\left(p_{1} p_{3}\right) \varphi+\left(p_{1} p_{2}\right) \kappa_{1}+p_{2}^{2} \kappa_{2}\right.\right. \\
& \left.+\left(p_{2} p_{3}\right) \kappa_{3}\right]-2 p_{2 \mu} p_{2 \mu_{3}}\left[p_{1}^{2}\left(p_{2} p_{3}\right) \varphi+p_{1}^{2} \kappa_{1}+\left(p_{1} p_{2}\right) \kappa_{2}+\left(p_{1} p_{3}\right) \kappa_{3}\right]+p_{1 \mu} p_{2 \mu_{3}}\left\{-\left[p_{1}^{2}\left(p_{2} p_{3}\right)\right.\right. \\
& \left.\left.+p_{2}^{2}\left(p_{1} p_{3}\right)\right] \varphi+\left(p_{1} p_{3}\right) \kappa_{1}+\left(p_{2} p_{3}\right) \kappa_{2}+p_{3}^{2} \kappa_{3}\right\}+p_{2 \mu} p_{1 \mu_{3}}\left\{\left[p_{2}^{2}\left(p_{1} p_{3}\right)+2\left(p_{1} p_{2}\right)\left(p_{2} p_{3}\right)\right] \varphi+3\left(p_{1} p_{2}\right) \kappa_{1}\right. \\
& \left.\left.+3 p_{2}^{2} \kappa_{2}+3\left(p_{2} p_{3}\right) \kappa_{3}\right\}\right) .
\end{aligned}
$$

For the scalar functions (2.10), Eq. (D1) yields

$$
\begin{gathered}
a^{(1,0)}\left(p_{3}, p_{2}, p_{1}\right)=-\frac{g^{2} \eta}{(4 \pi)^{n / 2}} C_{A} \frac{1}{4}\left\{\left(2 p_{1}^{2}-p_{2}^{2}\right) \varphi+\kappa_{1}-2 \kappa_{2}-\kappa_{3}\right\}, \\
b^{(1,0)}\left(p_{3}, p_{2}, p_{1}\right)=\frac{g^{2} \eta}{(4 \pi)^{n / 2}} C_{A} \frac{1}{2 \mathcal{K}}\left\{p_{1}^{2}\left(p_{2} p_{3}\right) \varphi+p_{1}^{2} \kappa_{1}+\left(p_{1} p_{2}\right) \kappa_{2}+\left(p_{1} p_{3}\right) \kappa_{3}\right\}, \\
c^{(1,0)}\left(p_{3}, p_{2}, p_{1}\right)=-\frac{g^{2} \eta}{(4 \pi)^{n / 2}} C_{A} \frac{1}{4 \mathcal{K}}\left\{\left(p_{1}^{2}-p_{2}^{2}\right)\left(p_{1} p_{2}\right) \varphi-\left[p_{1}^{2}-\left(p_{1} p_{2}\right)\right] \kappa_{1}+\left[p_{2}^{2}-\left(p_{1} p_{2}\right)\right] \kappa_{2}+\left(p_{1}^{2}-p_{2}^{2}\right) \kappa_{3}\right\}, \\
d^{(1,0)}\left(p_{3}, p_{2}, p_{1}\right)=\frac{g^{2} \eta}{(4 \pi)^{n / 2}} C_{A} \frac{1}{4 \mathcal{K}}\left\{\left[p_{2}^{2}\left(p_{1} p_{3}\right)+2\left(p_{1} p_{2}\right)\left(p_{2} p_{3}\right)\right] \varphi+3\left(p_{1} p_{2}\right) \kappa_{1}+3 p_{2}^{2} \kappa_{2}+3\left(p_{2} p_{3}\right) \kappa_{3}\right\}, \\
e^{(1,0)}\left(p_{3}, p_{2}, p_{1}\right)=-\frac{g^{2} \eta}{(4 \pi)^{n / 2}} C_{A} \frac{1}{4 \mathcal{K}}\left\{p_{3}^{2}\left(p_{1} p_{2}\right) \varphi+\left(p_{1} p_{3}\right) \kappa_{1}+\left(p_{2} p_{3}\right) \kappa_{2}+p_{3}^{2} \kappa_{3}\right\} .
\end{gathered}
$$

In the limit $n \rightarrow 4$, the expressions for all scalar functions (D2)-(D6) have been compared with the corresponding results presented in [13], taking into account the erratum (which affects the results for the $c, d$, and $e$ functions). The comparison was successful, with the exception of two minor things. One of them is related to the definition of the renormalization-scheme constant and was already mentioned before; see footnote in Sec. III A. The second one is that in the erratum [13] the sign of the term $b\left(P_{1}, P_{2}, P_{3}\right)$ in the expression for the $d$ function is changed from minus to plus (see p. 2554 of [13]). However, the comparison is successful if we keep the original sign, which is minus. ${ }^{26}$

Furthermore, the results which follow from Eq. (D1) (contracted with $p_{1}^{\mu}$ ) for two infrared-divergent cases, (i) $p_{1}^{2}=p_{2}^{2}=0$ and (ii) $p_{2}^{2}=p_{3}^{2}=0$ have been compared with those presented in Table B.II of Ref. [15]. The latter were obtained in the Feynman gauge and expanded in the limit $n \rightarrow 4$, keeping the finite [in $\varepsilon=(4-n) / 2]$ terms. To consider the limit of our expressions, we used formulas presented in Sec. IV C. We found that our results coincide in this limit with those from [15].

Now, we present the results for the scalar functions (2.10) in arbitrary covariant gauge:

$$
\begin{aligned}
a^{(1, \xi)}\left(p_{3}, p_{2}, p_{1}\right)= & -\frac{g^{2} \eta}{(4 \pi)^{n / 2}} C_{A} \frac{1}{16 \mathcal{K}}\left(\left\{2 \mathcal{K}[\xi(2 n-5)+2]+\xi[(n-3) \xi+2] p_{2}^{2}\left(p_{1} p_{3}\right)-\xi(\xi-2) p_{3}^{2}\left(p_{1} p_{2}\right)\right\}\left[\left(p_{2} p_{3}\right) \varphi+\kappa_{1}\right]\right. \\
& +\left\{(\xi-2)\left(4 \mathcal{K}-\xi p_{2}^{2} p_{3}^{2}\right)+\xi[(n-3) \xi+2] p_{2}^{2}\left(p_{2} p_{3}\right)\right\}\left[\left(p_{1} p_{3}\right) \varphi+\kappa_{2}\right]+\left\{(\xi-2)\left[(\xi+2) \mathcal{K}-\xi p_{3}^{2}\left(p_{2} p_{3}\right)\right]\right. \\
& \left.+\xi[(n-3) \xi+2]\left(p_{2} p_{3}\right)^{2}\right\}\left[\left(p_{1} p_{2}\right) \varphi+\kappa_{3}\right]+\xi\left(p_{2} p_{3}\right) \varphi\left\{(\xi-2)\left[\mathcal{K}+2 p_{3}^{2}\left(p_{1} p_{2}\right)\right]\right. \\
& \left.\left.+[(n-3) \xi+2]\left[\mathcal{K}-2\left(p_{1} p_{2}\right)\left(p_{2} p_{3}\right)\right]\right\}\right)
\end{aligned}
$$

\footnotetext{
${ }^{26}$ These misprints were confirmed by the authors of [13].
} 


$$
\begin{aligned}
b^{(1, \xi)}\left(p_{3}, p_{2}, p_{1}\right)= & -\frac{g^{2} \eta}{(4 \pi)^{n / 2}} C_{A} \frac{1}{16 \mathcal{K}^{2} p_{3}^{2}}\left[\left(\xi[(n-4) \xi+4](n-3)\left(p_{1} p_{2}\right)\left(p_{2} p_{3}\right) \mathcal{K}+[(n-3) \xi+2] p_{1}^{2} p_{3}^{2}[2(\xi-2) \mathcal{K}\right.\right. \\
& \left.\left.-\xi(n-1)\left(p_{1} p_{2}\right)\left(p_{2} p_{3}\right)\right]+\xi(\xi-2) p_{3}^{2}\left\{(n-1) p_{1}^{2} p_{3}^{2}\left(p_{1} p_{2}\right)+\mathcal{K}\left[p_{1}^{2}-2(n-3)\left(p_{1} p_{2}\right)\right]\right\}\right)\left[\left(p_{2} p_{3}\right) \varphi+\kappa_{1}\right] \\
& +\left(-\xi[(n-4) \xi+4](n-3)\left(p_{1} p_{2}\right)\left(p_{2} p_{3}\right) \mathcal{K}+\xi(\xi-2) p_{3}^{2}\left[(n-1) p_{1}^{2} p_{2}^{2} p_{3}^{2}+(n-2) \mathcal{K} \mathcal{Q}\right]\right. \\
& \left.+[(n-3) \xi+2] p_{3}^{2}\left\{\mathcal{K}\left[\xi p_{2}^{2}+(3 \xi-4)\left(p_{1} p_{2}\right)\right]-\xi(n-1)\left(p_{1} p_{2}\right)^{2}\left(p_{2} p_{3}\right)\right\}\right)\left[\left(p_{1} p_{3}\right) \varphi+\kappa_{2}\right] \\
& +\left(-\xi[(n-4) \xi+4](n-4)\left(p_{1} p_{3}\right)\left(p_{2} p_{3}\right) \mathcal{K}+[(n-3) \xi+2] p_{3}^{2}\left\{\xi\left(p_{2} p_{3}\right)\left[\mathcal{K}-(n-1)\left(p_{1} p_{2}\right)\left(p_{1} p_{3}\right)\right]\right.\right. \\
& \left.\left.+2(\xi-2) \mathcal{K}\left(p_{1} p_{3}\right)\right\}+\xi(\xi-2) p_{3}^{2}\left\{(n-1)\left(p_{2} p_{3}\right)\left(p_{1} p_{3}\right)^{2}-\mathcal{K}\left[p_{2}^{2}+\left(p_{1} p_{3}\right)\right]\right\}\right)\left[\left(p_{1} p_{2}\right) \varphi+\kappa_{3}\right] \\
& +\varphi\left(2 \xi[(n-4) \xi+4](n-3)\left(p_{1} p_{2}\right)\left(p_{1} p_{3}\right)\left(p_{2} p_{3}\right) \mathcal{K}+[(n-3) \xi+2] p_{3}^{2}\left\{2 \xi(n-1)\left(p_{1} p_{2}\right)^{2}\left(p_{1} p_{3}\right)\right.\right. \\
& \left.\times\left(p_{2} p_{3}\right)-2 \mathcal{K}\left(p_{1} p_{3}\right)\left[2(\xi-2)\left(p_{1} p_{2}\right)+\xi p_{2}^{2}\right]-\xi \mathcal{K}^{2}\right\}+\xi(\xi-2) p_{3}^{2}\left[2(n-2) \mathcal{K}\left(p_{1} p_{2}\right)\left(p_{2} p_{3}\right)\right. \\
& \left.\left.\left.-2(n-1) p_{1}^{2} p_{3}^{2}\left(p_{1} p_{2}\right)\left(p_{2} p_{3}\right)+\mathcal{K}^{2}\right]\right)\right],
\end{aligned}
$$

$$
\begin{aligned}
c^{(1, \xi)}\left(p_{3}, p_{2}, p_{1}\right)= & -\frac{g^{2} \eta}{(4 \pi)^{n / 2}} C_{A} \frac{1}{16 \mathcal{K}^{2} p_{1}^{2}}\left\{\left[[ ( n - 3 ) \xi + 2 ] \left(\xi(n-1)\left(p_{1} p_{2}\right)^{2}\left(p_{1} p_{3}\right)^{2}-\mathcal{K}\left\{4\left(p_{1}^{2}\right)^{2}+\left(p_{1} p_{3}\right)\left[(\xi+2) p_{1}^{2}\right.\right.\right.\right.\right. \\
& \left.\left.\left.\left.+3 \xi\left(p_{1} p_{2}\right)\right]\right\}\right)-\xi(\xi-2)\left(p_{1} p_{3}\right)\left\{(n-1)\left(p_{1} p_{2}\right)\left(p_{1} p_{3}\right)^{2}+\mathcal{K}\left[2 p_{1}^{2}+3\left(p_{1} p_{2}\right)\right]\right\}\right]\left[\left(p_{2} p_{3}\right) \varphi+\kappa_{1}\right] \\
& +\left([(n-3) \xi+2]\left\{\xi\left(p_{1} p_{3}\right)\left(p_{2} p_{3}\right)\left[(n-1)\left(p_{1} p_{2}\right)^{2}+2 \mathcal{K}\right]+2 \mathcal{K} p_{1}^{2}\left[p_{2}^{2}-\left(p_{1} p_{2}\right)\right]\right\}+\xi(\xi-2)\left(p_{1} p_{3}\right)\right. \\
& \left.\times\left[p_{1}^{2} \mathcal{K}-(n-3) \mathcal{K} \mathcal{Q}-(n-1) p_{1}^{2} p_{2}^{2} p_{3}^{2}\right]\right)\left[\left(p_{1} p_{3}\right) \varphi+\kappa_{2}\right]+\left[[ ( n - 3 ) \xi + 2 ] \left(\xi(n-1)\left(p_{1} p_{2}\right)\right.\right. \\
& \left.\times\left(p_{1} p_{3}\right)^{2}\left(p_{2} p_{3}\right)-\mathcal{K}\left\{(\xi-2) p_{1}^{2}\left(p_{1}^{2}-p_{2}^{2}\right)+\xi\left(p_{2} p_{3}\right)\left[2\left(p_{1} p_{3}\right)-p_{1}^{2}\right]\right\}\right)+\xi(\xi-2)\left(p_{1} p_{3}\right)\left(p_{2} p_{3}\right)[(n-3) \mathcal{K} \\
& \left.\left.-(n-1) p_{1}^{2} p_{3}^{2}\right]\right]\left[\left(p_{1} p_{2}\right) \varphi+\kappa_{3}\right]+2 \varphi\left\{-[(n-3) \xi+2]\left(p_{1} p_{2}\right)\left[2 p_{1}^{2}\left(p_{1}^{2}-p_{2}^{2}\right) \mathcal{K}+\xi(n-1)\left(p_{1} p_{2}\right)\right.\right. \\
& \left.\left.\left.\times\left(p_{1} p_{3}\right)^{2}\left(p_{2} p_{3}\right)\right]+\xi\left(p_{1} p_{3}\right)\left(p_{2} p_{3}\right)\left[(\xi-2)(n-1)\left(p_{1} p_{2}\right)\left(p_{1} p_{3}\right)^{2}+\xi(n-2) \mathcal{K}\left(p_{3}^{2}-p_{2}^{2}\right)\right]\right\}\right\},
\end{aligned}
$$

$$
\begin{aligned}
d^{(1, \xi)}\left(p_{3}, p_{2}, p_{1}\right)= & -\frac{g^{2} \eta}{(4 \pi)^{n / 2}} C_{A} \frac{1}{16 \mathcal{K}^{2} p_{3}^{2}}\left[\left(\xi[(n-4) \xi+4]\left(p_{1} p_{2}\right)\left(p_{1} p_{3}\right)\left[(n-3)\left(p_{1} p_{3}\right)\left(p_{2} p_{3}\right)+p_{3}^{2}\left(p_{1} p_{2}\right)\right]\right.\right. \\
& -[(n-3) \xi+2] p_{3}^{2}\left\{\xi\left(p_{2} p_{3}\right)\left[p_{1}^{2} p_{2}^{2}-(n-2)\left(p_{1} p_{2}\right)\left(p_{1} p_{3}\right)\right]+6\left(p_{1} p_{2}\right) \mathcal{K}\right\}+\xi(\xi-2) p_{3}^{2}\left(p_{2} p_{3}\right)\left[p_{1}^{2}\left(p_{2} p_{3}\right)\right. \\
& \left.\left.-(n-2)\left(p_{1} p_{2}\right)^{2}\right]\right)\left[\left(p_{2} p_{3}\right) \varphi+\kappa_{1}\right]+\left(\xi[(n-4) \xi+4](n-3)\left(p_{1} p_{2}\right)\left(p_{1} p_{3}\right)\left(p_{2} p_{3}\right)^{2}\right. \\
& +[(n-3) \xi+2] p_{3}^{2}\left\{\xi\left(p_{1} p_{2}\right)\left(p_{2} p_{3}\right)\left[(n-3)\left(p_{2} p_{3}\right)-2 p_{2}^{2}\right]-4 p_{2}^{2} \mathcal{K}\right\}-(\xi-2) p_{3}^{2}\left\{\xi\left(p_{2} p_{3}\right)\right. \\
& \left.\left.\times\left[p_{1}^{2} p_{2}^{2}+(n-1) p_{2}^{2}\left(p_{1} p_{2}\right)+\left(p_{1} p_{2}\right)^{2}\right]+(\xi-2) p_{2}^{2} \mathcal{K}\right\}\right)\left[\left(p_{1} p_{3}\right) \varphi+\kappa_{2}\right]+\left\{\xi[(n-4) \xi+4](n-4)\left(p_{1} p_{3}\right)\right. \\
& \times\left(p_{2} p_{3}\right) \mathcal{K}+\xi(\xi-2) p_{3}^{2}\left(p_{1} p_{2}\right)\left[(n-1) p_{3}^{2}\left(p_{2} p_{3}\right)-\mathcal{K}\right]-[(n-3) \xi+2] p_{3}^{2}\left(p_{2} p_{3}\right)\left[\xi(n-1) p_{2}^{2}\left(p_{1} p_{3}\right)\right. \\
& +(\xi+6) \mathcal{K}]\}\left[\left(p_{1} p_{2}\right) \varphi+\kappa_{3}\right]+2 \varphi\left(-[(n-4) \xi+4]\left(p_{1} p_{2}\right)\left(p_{2} p_{3}\right) \mathcal{K}\left[\xi(n-3)\left(p_{1} p_{3}\right)+(\xi-2) p_{3}^{2}\right]\right. \\
& +[(n-3) \xi+2] p_{3}^{2}\left\{\xi\left(p_{1} p_{2}\right)\left(p_{2} p_{3}\right)\left[2 \mathcal{K}+n p_{2}^{2}\left(p_{1} p_{3}\right)\right]-2\left(p_{2}^{2}\right)^{2}\left(p_{1} p_{3}\right)^{2}\right\}+(\xi-2) p_{3}^{2}\left(p_{1} p_{2}\right)\left(p_{1} p_{3}\right)\left(p_{2} p_{3}\right) \\
& \left.\left.\times\left[\xi(n-1)\left(p_{1} p_{2}\right)+2(\xi-1) p_{2}^{2}\right]\right)\right],
\end{aligned}
$$

$$
\begin{aligned}
e^{(1, \xi)}\left(p_{3}, p_{2}, p_{1}\right)= & -\frac{g^{2} \eta}{(4 \pi)^{n / 2}} C_{A} \frac{1}{16 \mathcal{K}^{2} p_{1}^{2}}\left[\left([(n-3) \xi+2]\left\{-\xi(n-1) p_{3}^{2}\left(p_{1} p_{2}\right)^{2}\left(p_{1} p_{3}\right)+\mathcal{K} p_{1}^{2}\left[2\left(p_{1} p_{3}\right)-\xi p_{3}^{2}\right]\right\}\right.\right. \\
& \left.+\xi(\xi-2)(n-1) p_{3}^{2}\left(p_{1} p_{2}\right)\left(p_{1} p_{3}\right)^{2}+\xi^{2}(n-2) \mathcal{K} p_{3}^{2}\left[2 p_{1}^{2}+3\left(p_{1} p_{2}\right)\right]\right)\left[\left(p_{2} p_{3}\right) \varphi+\kappa_{1}\right]+([(n-3) \xi+2] \\
& \times\left(p_{2} p_{3}\right)\left\{-\xi(n-1) p_{1}^{2} p_{2}^{2} p_{3}^{2}+\mathcal{K}\left[\xi(n-3) p_{3}^{2}+2 p_{1}^{2}\right]\right\}+\xi(\xi-2) p_{3}^{2}\left\{(n-1) p_{1}^{2} p_{2}^{2} p_{3}^{2}+\mathcal{K}[(n-3) \mathcal{Q}\right. \\
& \left.\left.\left.-p_{1}^{2}\right]\right\}\right)\left[\left(p_{1} p_{3}\right) \varphi+\kappa_{2}\right]+\left([(n-3) \xi+2]\left\{-\xi(n-1) p_{1}^{2} p_{2}^{2} p_{3}^{2}+\mathcal{K}\left[(\xi+2) p_{1}^{2}-(n-3) \xi \mathcal{Q}\right]\right\}+\xi(\xi-2)\left(p_{2} p_{3}\right)\right. \\
& \left.\times\left[(n-1)\left(p_{1} p_{3}\right)^{2}+2 \mathcal{K}\right]\right) p_{3}^{2}\left[\left(p_{1} p_{2}\right) \varphi+\kappa_{3}\right]+2\left(p_{2} p_{3}\right) \varphi\left\{[ ( n - 3 ) \xi + 2 ] ( p _ { 1 } p _ { 3 } ) \left[\xi(n-1) p_{3}^{2}\left(p_{1} p_{2}\right)^{2}\right.\right. \\
& \left.\left.\left.-2 \mathcal{K} p_{1}^{2}\right]-\xi(\xi-2)(n-1) p_{3}^{2}\left(p_{1} p_{2}\right)\left(p_{1} p_{3}\right)^{2}-\xi^{2}(n-2) \mathcal{K} p_{3}^{2}\left(p_{3}^{2}-p_{2}^{2}\right)\right\}\right] .
\end{aligned}
$$


From the expressions (D7)-(D11), one can see that again, as in the three-gluon functions (3.10) $-(3.15)$, the values $\xi=0$ and $\xi=-4 /(n-4)$ are distinguished. Putting $\xi=0$, we get rid of the momenta squared in the denominators [only $\mathcal{K}$ may remain; cf. Eqs. (D2)-(D6)], while for $\xi=-4 /(n-4)$ one of the momenta squared, $p_{1}^{2}$, still survives in the denominators of the $c$ function, Eq. (D9), and the $e$ function, Eq. (D11). This does not matter, since (i) the $c$ and $e$ functions do not contribute to the Ward-Slavnov-Taylor identity for the three-gluon vertex, Eq. (2.12), and (ii) for the proper ghost-gluon vertex, Eq. (2.9), we get an extra $p_{1}^{2}$ in the numerator as a result of contracting with $p_{1}{ }^{\mu}$ [since the tensor structures corresponding to the $c$ and $e$ functions contain $p_{1 \mu}$; see Eq. (2.10)]. If one wants to put some of the momenta squared equal to zero in other gauges, one should carefully consider the appropriate limit and expand the functions in the numerator, in exactly the same way as it was described in Sec. IV for the case of the three-gluon vertex.

In the limit $n \rightarrow 4$, the only divergent function in the one-loop ghost-gluon vertex is the $a$ function (D7), also since this is the only function which is present at the zero-loop level, Eq. (2.11). The ultraviolet-divergent part of the $a$ function (D7) is

$$
a^{(1, \mathrm{UV})}=\frac{g^{2} \eta}{(4 \pi)^{2-\varepsilon}} C_{A} \frac{1}{2}(1-\xi) \kappa^{(\mathrm{UV})},
$$

where, as in Eq. (3.37), $\kappa^{(\mathrm{UV})}=1 / \varepsilon+\cdots$ corresponds to the divergent part of the function $\kappa$, Eq. (2.15). Therefore, by analogy with (3.38), the counterterm contribution is given by ${ }^{27}$

$$
a^{(1, \mathrm{CT})}=-\frac{\vec{g}^{2}}{(4 \pi)^{2}} \frac{C_{A}}{2}(1-\xi)\left(\frac{1}{\varepsilon}+R\right)=-\frac{g^{2} \eta}{(4 \pi)^{2-\varepsilon}} \frac{C_{A}}{2}(1-\xi)\left(\frac{1}{\varepsilon}+R\right)+O(\varepsilon),
$$

where $\vec{g}^{2} \equiv g^{2} e^{-\gamma \varepsilon}(4 \pi)^{\varepsilon}$ and $R$ is the renormalization-scheme constant $[R=0$ in the $\overline{\text { MS }}$ scheme, which is achieved by a suitable extraction of the overall factor in Eq. (D13)]. In particular, there is no singularity in the one-loop ghost-gluon vertex in the Landau gauge $(\xi=1)$; see, e.g., Ref. [2].

\section{APPENDIX E: RESULTS FOR $p_{3}^{2}=0$ IN ARBITRARY GAUGE}

For arbitrary $\xi$, the results for the gluon and ghost loop contributions to the scalar functions $(2.4)$ are $^{28}$

$$
\begin{aligned}
& A^{(1, \xi)}\left(p_{1}^{2}, p_{2}^{2} ; 0\right)=-\frac{g^{2} \eta}{(4 \pi)^{n / 2}} C_{A} \frac{1}{64(n-1)(n-4)}\left\{(n-1)(n-4) \xi[4+(n-4) \xi]\left[\frac{p_{2}^{2}}{p_{1}^{2}} \kappa_{1}+\frac{p_{1}^{2}}{p_{2}^{2}} \kappa_{2}\right]\right. \\
& +(n-4)\left[16(3 n-2)+4 \xi(n-1)(10 n-33)-5 \xi^{2}(n-1)(n-4)\right]\left[\kappa_{1}+\kappa_{2}\right]-16(n-1) \\
& \left.\times[n+(n-3) \xi]\left(\delta_{12}\right)^{-1}\left[\kappa_{1}-\kappa_{2}\right]\right\}, \\
& A^{(1, \xi)}\left(0, p_{1}^{2} ; p_{2}^{2}\right)=-\frac{g^{2} \eta}{(4 \pi)^{n / 2}} C_{A} \frac{1}{64(n-1)(n-4)(n-6)}\left\{\frac{p_{2}^{2}}{p_{1}^{2}} \kappa_{1}(n-1)(n-4)(n-6) \xi[4+(n-4) \xi]+\kappa_{1}(n-4)(n-6)\right. \\
& \times\left[16(3 n-2)+4 \xi(n-1)(10 n-33)-5 \xi^{2}(n-1)(n-4)\right]+2 \kappa_{2}(n-1)(n-4)(n-6) \xi[4(n-3) \\
& -(n-4) \xi]-8(n-1)(n-4) \kappa_{2}\left\{\left(\delta_{12}\right)^{-1}(n-6)[2+(n-3) \xi]+\left(\delta_{12}\right)^{-2} \xi[n+(n-3) \xi]\right\}-4(n-1) \\
& \times\left[\kappa_{1}-\kappa_{2}\right]\left\{\left(\delta_{12}\right)^{-1}(n-6)\left[4(n+1)+\xi(5 n-18)-\xi^{2}(n-3)\right]+\left(\delta_{12}\right)^{-2}[4+(n-4) \xi][(n-3) \xi-n+6]\right. \\
& \left.\left.-2\left(\delta_{12}\right)^{-3} \xi[n+(n-3) \xi]\right\}\right\} \\
& B^{(1, \xi)}\left(p_{1}^{2}, p_{2}^{2} ; 0\right)=-\frac{g^{2} \eta}{(4 \pi)^{n / 2}} C_{A} \frac{1}{64(n-1)(n-4)(n-6)}\left\{(n-1)(n-4)(n-6) \xi[4+(n-4) \xi]\left[\frac{p_{2}^{2}}{p_{1}^{2}} \kappa_{1}-\frac{p_{1}^{2}}{p_{2}^{2}} \kappa_{2}\right]\right. \\
& -8(n-1)(n-4)\left[\kappa_{1}+\kappa_{2}\right]\left(\delta_{12}\right)^{-1} \xi[n+(n-3) \xi]+\left[\kappa_{1}-\kappa_{2}\right]\left\{16(n-6)\left(4 n^{2}-21 n+14\right)\right. \\
& +4 \xi(n-1)(n-6)\left(10 n^{2}-77 n+152\right)-\xi^{2}(n-1)(n-6)\left(5 n^{2}-48 n+104\right)+16\left(\delta_{12}\right)^{-2} \\
& \times(n-1) \xi[n+(n-3) \xi]\},
\end{aligned}
$$

\footnotetext{
${ }^{27}$ See also the discussion of $\mu_{\mathrm{DR}}, \overrightarrow{g^{2}}$, etc., in Sec. III E.

${ }^{28} \mathrm{We}$ also consider nonequivalent permutations of the arguments.
} 


$$
\begin{aligned}
B^{(1, \xi)}\left(0, p_{1}^{2} ; p_{2}^{2}\right)= & -\frac{g^{2} \eta}{(4 \pi)^{n / 2}} C_{A} \frac{1}{64(n-1)(n-4)(n-6)}\left\{-(n-1)(n-4)(n-6) \xi[4+(n-4) \xi]\left[\frac{p_{2}^{2}}{p_{1}^{2}} \kappa_{1}+2 \frac{p_{1}^{2}}{p_{2}^{2}} \kappa_{2}\right]-8(n-1)\right. \\
& \times(n-4) \kappa_{2}\left\{(n-6)[2+(n-3) \xi]\left[1+\left(\delta_{12}\right)^{-1}\right]+\left(\delta_{12}\right)^{-2} \xi[n+(n-3) \xi]\right\}-(n-4)(n-6) \kappa_{1}[16(4 n-3) \\
& \left.+20 \xi(n-1)(2 n-7)-5 \xi^{2}(n-1)(n-4)\right]+4(n-1)\left[\kappa_{1}-\kappa_{2}\right]\left\{\left(\delta_{12}\right)^{-1}(n-6)\left[4+3 \xi(n-2)+\xi^{2}(n-3)\right]\right. \\
& \left.\left.-\left(\delta_{12}\right)^{-2}[4+(n-4) \xi][(n-3) \xi-n+6]+2\left(\delta_{12}\right)^{-3} \xi[n+(n-3) \xi]\right\}\right\},
\end{aligned}
$$

$$
\begin{aligned}
C^{(1, \xi)}\left(p_{1}^{2}, p_{2}^{2} ; 0\right)= & -\frac{g^{2} \eta}{(4 \pi)^{n / 2}} C_{A} \frac{1}{32(n-1)(n-4)\left(p_{1}^{2}-p_{2}^{2}\right)}\left\{\left[\frac{p_{2}^{2}}{p_{1}^{2}} \kappa_{1}-\frac{p_{1}^{2}}{p_{2}^{2}} \kappa_{2}\right](n-1)(n-4) \xi[4+(n-4) \xi]+\left[\kappa_{1}-\kappa_{2}\right]\right. \\
& \left.\times\left[16 n(n-4)+4 \xi(n-1)\left(6 n^{2}-41 n+72\right)-3 \xi^{2}(n-1)(n-4)^{2}\right]\right\}
\end{aligned}
$$

$$
\begin{aligned}
& C^{(1, \xi)}\left(0, p_{1}^{2} ; p_{2}^{2}\right)=-\frac{g^{2} \eta}{(4 \pi)^{n / 2}} C_{A} \frac{1}{32(n-1)(n-4)(n-6) p_{1}^{2}\left(p_{1}^{2}-p_{2}^{2}\right)}\left\{8(n-1) p_{1}^{2}\left[\kappa_{1}-\kappa_{2}\right]\{-(n-6)[6+(n-5) \xi]\right. \\
& \left.+(n-6)\left(\delta_{12}\right)^{-1}\left[6+\xi(n-7)-\xi^{2}(n-3)\right]-2\left(\delta_{12}\right)^{-2} \xi[n+(n-3) \xi]\right\}+8(n-1)(n-4)\left(\delta_{12}\right)^{-1} \\
& \times\left(p_{1}^{2}+p_{2}^{2}\right) \kappa_{1} \xi[n+(n-3) \xi]+(n-4)(n-6) \kappa_{1}\left[-\frac{\left(p_{2}^{2}\right)^{2}}{p_{1}^{2}}(n-1) \xi[4+(n-4) \xi]+16\left[n p_{1}^{2}-(4 n-3) p_{2}^{2}\right]\right. \\
& \left.\left.+2 \xi(n-1)\left[(12 n-38) p_{1}^{2}-4(5 n-17) p_{2}^{2}\right]+\xi^{2}(n-1)(n-4)\left(-3 p_{1}^{2}+4 p_{2}^{2}\right)\right]\right\} \\
& F^{(1, \xi)}\left(p_{1}^{2}, p_{2}^{2}, 0\right)=-\frac{g^{2} \eta}{(4 \pi)^{n / 2}} C_{A} \frac{1}{16(n-1)(n-4)(n-6)\left(p_{1}^{2}-p_{2}^{2}\right)}\left\{\left[\frac{1}{p_{1}^{2}} \kappa_{1}-\frac{1}{p_{2}^{2}} \kappa_{2}\right](n-1)(n-4) \xi[4+(n-4) \xi]\right. \\
& \times[4 \xi(n-3)-5(n-6)]+\frac{2}{p_{1}^{2}-p_{2}^{2}}\left\{(n-4)\left[\kappa_{1}+\kappa_{2}\right]-2\left(\delta_{12}\right)^{-1}\left[\kappa_{1}-\kappa_{2}\right]\right\}[8(n-6)(4 n-7) \\
& \left.\left.+4 \xi(n-1)(n-6)(5 n-11)+\xi^{2}(n-1)\left(5 n^{2}-60 n+108\right)-2 \xi^{3} n(n-1)(n-3)\right]\right\},
\end{aligned}
$$

$$
\begin{aligned}
& F^{(1, \xi)}\left(0, p_{1}^{2} ; p_{2}^{2}\right)=-\frac{g^{2} \eta}{(4 \pi)^{n / 2}} C_{A} \frac{1}{32(n-1)(n-4)(n-6) p_{1}^{2}\left(p_{1}^{2}-p_{2}^{2}\right)}\left\{-\frac{2}{p_{1}^{2}-p_{2}^{2}}\left[(n-6) \frac{\left(p_{2}^{2}\right)^{2}}{p_{1}^{2}} \kappa_{1}+4(n-3) \xi \frac{\left(p_{1}^{2}\right)^{2}}{p_{2}^{2}} \kappa_{2}\right](n-1)\right. \\
& \times(n-4) \xi[4+(n-4) \xi]+(n-4) \kappa_{1}\left\{\left[32(n-6)(3 n+1)+4 \xi(n-1)(n-6)(20 n-63)-3 \xi^{2}(n-1)(n-4)\right.\right. \\
& \left.\times(n+2)-4 \xi^{3}(n-1)(n-3)(n-4)\right]-\left(\delta_{12}\right)^{-1}\left[32(n-6)(n+2)+4 \xi(n-1)(7 n+30)+\xi^{2}(n-1)\right. \\
& \left.\times\left(3 n^{2}+50 n-24\right)-4 \xi^{3}(n-1)(n-3)(n-16)\right]-24\left(\delta_{12}\right)^{-2}\left[8(n-6)+8 \xi(n-1)+\xi^{2}(n-1)\right. \\
& \left.\left.\times(3 n-2)+2 \xi^{3}(n-1)(n-3)\right]\right\}+16 p_{1}^{2} \frac{\kappa_{1}-\kappa_{2}}{p_{1}^{2}-p_{2}^{2}}\left\{\left[4(n-6)\left(n^{2}-12 n+17\right)-2 \xi(n-1)(n-6)(3 n-10)\right.\right. \\
& \left.+\xi^{2}(n-1)(n-5)\left(n^{2}-6 n+12\right)+2 \xi^{3}(n-1)(n-3)(n-5)\right]+\left(\delta_{12}\right)^{-1}[16(n-4)(n-6)+4 \xi(n-1) \\
& \left.\times(4 n-15)+\xi^{2}(n-1)(n-4)(5 n-3)+3 \xi^{3}(n-1)(n-3)(n-4)\right]+3\left(\delta_{12}\right)^{-2}[8(n-6)+8 \xi(n-1) \\
& \left.\left.\left.+\xi^{2}(n-1)(3 n-2)+2 \xi^{3}(n-1)(n-3)\right]\right\}\right\},
\end{aligned}
$$




$$
\begin{aligned}
H^{(1, \xi)}\left(p_{1}^{2}, p_{2}^{2}, 0\right)= & -\frac{g^{2} \eta}{(4 \pi)^{n / 2}} C_{A} \frac{1}{16(n-1)(n-4)(n-6)\left(p_{1}^{2}-p_{2}^{2}\right)}\left\{-\left[\frac{p_{2}^{2}}{p_{1}^{2}} \kappa_{1}-\frac{p_{1}^{2}}{p_{2}^{2}} \kappa_{2}\right](n-1)(n-4) \xi[4+(n-4) \xi][2 \xi(n-3)\right. \\
& -3(n-6)]+2\left(\delta_{12}\right)^{-1}\left\{(n-4)\left[\kappa_{1}+\kappa_{2}\right]-2\left(\delta_{12}\right)^{-1}\left[\kappa_{1}-\kappa_{2}\right]\right\}\left[24(n-6)+2 \xi(n-1)(n+12)+\xi^{2}(n-1)\right. \\
& \left.\times(11 n-12)+6 \xi^{3}(n-1)(n-3)\right]+\left[\kappa_{1}-\kappa_{2}\right]\left[16(n-6)\left(n^{2}-2 n-2\right)+4 \xi(n-1)(n-6)\left(6 n^{2}-37 n+60\right)\right. \\
& \left.\left.-\xi^{2}(n-1)\left(7 n^{3}-88 n^{2}+376 n-552\right)-8 \xi^{3}(n-1)(n-3)(n-5)\right]\right\},
\end{aligned}
$$

where $\kappa_{i} \equiv \kappa\left(p_{i}^{2}\right)$ [see Eq. (2.15)], while the coefficients $\eta$ and $C_{A}$ are defined by Eqs. (2.17) and (3.3), respectively.

\section{APPENDIX F: RESULTS FOR $p_{1}^{2}=p_{2}^{2}=0$ IN AN ARBITRARY GAUGE}

The scalar functions $U_{i}$ corresponding to the decomposition of the three-gluon vertex in this limit are defined by Eq. (4.58). Using the expressions for the one-loop contributions to the $A, B, C, F$, and $H$ functions, Eqs. (4.43)-(4.57), and the representations (4.59)-(4.65), we get the following results for the one-loop contributions to the $U_{i}$ functions:

$$
\begin{aligned}
& U_{1}^{(1, \xi)}\left(p^{2}\right)=-\frac{g^{2} \eta}{(4 \pi)^{n / 2}} C_{A} \frac{1}{8(n-1)(n-4)} \kappa\left\{4\left(2 n^{2}-15 n+19\right)+2 \xi(n-1)(n-3)(4 n-17)-\xi^{2}(n-1)(n-4)^{2}\right\}, \\
& U_{2}^{(1, \xi)}\left(p^{2}\right)=\frac{g^{2} \eta}{(4 \pi)^{n / 2}} C_{A} \frac{1}{4(n-1)(n-4)} \kappa\left\{2\left(3 n^{2}-17 n+8\right)+\xi(n-1)(n-4)(8 n-27)-\xi^{2}(n-1)(n-4)^{2}\right\}, \\
& U_{3}^{(1, \xi)}\left(p^{2}\right)=\frac{g^{2} \eta}{(4 \pi)^{n / 2}} C_{A} \frac{1}{16(n-1)(n-4)} \kappa\left\{4(n-4)(n-5)+2 \xi(n-1)\left(2 n^{2}-19 n+36\right)-\xi^{2}(n-1)\left(n^{2}-8 n+20\right)\right\}, \\
& U_{4}^{(1, \xi)}\left(p^{2}\right)=\frac{g^{2} \eta}{(4 \pi)^{n / 2}} C_{A} \frac{1}{4(n-1)(n-4)(n-6) p^{2}} \kappa\left\{6(n-2)(n-6)(n-7)+\xi(n-1)(n-6)\left(2 n^{2}-23 n+40\right)\right. \\
& \left.+\xi^{2}(n-1)(n-2)\left(n^{2}-15 n+57\right)-\xi^{3}(n-1)(n-3)(n-8)\right\}, \\
& U_{5}^{(1, \xi)}\left(p^{2}\right)=\frac{g^{2} \eta}{(4 \pi)^{n / 2}} C_{A} \frac{1}{4(n-1)(n-4) p^{2}} \kappa\left\{4(n-4)^{2}+\xi(n-1)(n-6)\right\}, \\
& U_{6}^{(1, \xi)}\left(p^{2}\right)=-\frac{g^{2} \eta}{(4 \pi)^{n / 2}} C_{A} \frac{1}{16(n-1)(n-4) p^{2}} \kappa\left\{16 n(n-4)+4 \xi(n-1)\left(6 n^{2}-41 n+72\right)-3 \xi^{2}(n-1)(n-4)^{2}\right\}, \\
& U_{7}^{(1, \xi)}\left(p^{2}\right)=-\frac{g^{2} \eta}{(4 \pi)^{n / 2}} C_{A} \frac{1}{16(n-1)(n-4) p^{2}} \kappa\left\{8\left(5 n^{2}-25 n+2\right)+4 \xi(n-1)\left(8 n^{2}-59 n+112\right)\right. \\
& \left.-\xi^{2}(n-1)\left(3 n^{2}-24 n+40\right)\right\} \text {, } \\
& U_{1}^{(1, q)}\left(p^{2}\right)=\frac{g^{2} \eta}{(4 \pi)^{n / 2}} 2 N_{f} T_{R} \frac{n(n-3)}{(n-1)(n-2)} \kappa, \\
& U_{2}^{(1, q)}\left(p^{2}\right)=-\frac{g^{2} \eta}{(4 \pi)^{n / 2}} 4 N_{f} T_{R} \frac{n-2}{n-1} \kappa, \\
& U_{3}^{(1, q)}\left(p^{2}\right)=\frac{g^{2} \eta}{(4 \pi)^{n / 2}} 4 N_{f} T_{R} \frac{1}{(n-1)(n-2)} \kappa, \\
& U_{4}^{(1, q)}\left(p^{2}\right)=\frac{g^{2} \eta}{(4 \pi)^{n / 2}} 4 N_{f} T_{R} \frac{n+2}{(n-1)(n-2) p^{2}} \kappa \\
& U_{5}^{(1, q)}\left(p^{2}\right)=-\frac{g^{2} \eta}{(4 \pi)^{n / 2}} 4 N_{f} T_{R} \frac{n-4}{(n-1)(n-2) p^{2}} \kappa,
\end{aligned}
$$




$$
\begin{gathered}
U_{6}^{(1, q)}\left(p^{2}\right)=\frac{g^{2} \eta}{(4 \pi)^{n / 2}} 4 N_{f} T_{R} \frac{n-2}{(n-1) p^{2}} \kappa, \\
U_{7}^{(1, q)}\left(p^{2}\right)=\frac{g^{2} \eta}{(4 \pi)^{n / 2}} 4 N_{f} T_{R} \frac{n^{2}-4 n+6}{(n-1)(n-2) p^{2}} \kappa,
\end{gathered}
$$

where, as usual, $\kappa \equiv \kappa\left(p^{2}\right)$. Comparison with the definition of the functions $F_{i}$ given in Eq. (29) of [14] shows that the functions $U_{1}, U_{2}, U_{3}, U_{4}, U_{5}, U_{6}$, and $U_{7}$ are proportional to $F_{2}, F_{3}, F_{1}, F_{6}, F_{5}, F_{4}$, and $F_{7}$, respectively.

[1] H. Fritzsch, M. Gell-Mann, and H. Leutwyler, Phys. Lett. B 47B, 365 (1973); D. J. Gross and F. Wilczek, Phys. Rev. Lett. 30, 1343 (1973); H. D. Politzer, ibid. 30, 1346 (1973); S. Weinberg, ibid. 31, 494 (1973).

[2] W. Marciano and H. Pagels, Phys. Rep. 36, 137 (1978).

[3] CTEQ Collaboration, R. Brock et al., Rev. Mod. Phys. 67, 157 (1995).

[4] S. Bethke, in Proceedings of the XXVIth International Conference on High Energy Physics, Dallas, Texas, 1992, edited by J. R. Sanford, AIP Conf. Proc. 272 (AIP, New York, 1993), p. 81; B. R. Webber, in Proceedings of the XXVIIth International Conference on High Energy Physics, Glasgow, Scotland, 1994, edited by P. J. Bussey and I. G. Knowles (IOP, London, 1995), p. 213.

[5] ALEPH Collaboration, D. Decamp et al., Phys. Lett. B 284, 151 (1992); DELPHI Collaboration, P. Abreu et al., Z. Phys. C 59, 357 (1993); OPAL Collaboration, R. Akers et al., ibid. 65, 367 (1995).

[6] C. Itzykson and J.-B. Zuber, Quantum Field Theory (McGrawHill, New York, 1980).

[7] P. Pascual and R. Tarrach, QCD: Renormalization for the Practitioner, Lecture Notes in Physics Vol. 194 (Springer, Berlin, 1984).

[8] T. Muta, Foundations of Quantum Chromodynamics (World Scientific, Singapore, 1987).

[9] W. Beenakker, H. Kuijf, W. L. van Neerven, and J. Smith, Phys. Rev. D 40, 54 (1989); W. T. Giele, E. W. N. Glover, and D. A. Kosower, Nucl. Phys. B403, 633 (1993); Z. Kunszt, A. Signer, and Z. Troscanyi, ibid. B411, 397 (1994); L. Dixon and Y. Shadmi, ibid. B423, 3 (1994); G. Mahlon, Phys. Rev. D 49, 2197 (1994); Z. Bern, L. Dixon, and D. A. Kosower, Nucl. Phys. B437, 259 (1995).

[10] B. A. Arbuzov, Sov. J. Part. Nucl. 19, 1 (1988).

[11] W. Celmaster and R. J. Gonsalves, Phys. Rev. D 20, 1420 (1979).

[12] P. Pascual and R. Tarrach, Nucl. Phys. B174, 123 (1980).

[13] J. S. Ball and T.-W. Chiu, Phys. Rev. D 22, 2550 (1980); 23, 3085(E) (1981).

[14] F. T. Brandt and J. Frenkel, Phys. Rev. D 33, 464 (1986).

[15] M. A. Nowak, M. Praszalowicz, and W. Slomiński, Ann. Phys. (N.Y.) 166, 443 (1986).

[16] M. Teper, Phys. Lett. B 289, 115 (1992).

[17] E. Abdalla and M. C. B. Abdalla, Phys. Rep. 265, 253 (1996).

[18] M. Dalbosco, Phys. Lett. 163B, 181 (1985); H. C. Lee, M. S. Milgram, and A. Andraši, Z. Phys. C 33, 107 (1986); A. Andraši, G. Leibbrandt, and S.-L. Nyeo, Nucl. Phys. B276, 445 (1986); A. T. Suzuki, Z. Phys. C 38, 595 (1988); E. Bagan and C. P. Martin, Nucl. Phys. B341, 419 (1990).
[19] D. Z. Friedman, G. Grignani, K. Johnson, and N. Rius, Ann. Phys. (N.Y.) 219, 75 (1992).

[20] J. M. Cornwall and J. Papavassiliou, Phys. Rev. D 40, 3474 (1989).

[21] C. Parrinello, Phys. Rev. D 50, 4247 (1994); D. Henty, C. Parrinello, and C. Pittori, talk given at International Europhysics Conference on High Energy Physics (HEP 95), Brussels, Belgium, 1995, Report No. hep-lat/9510045 (unpublished).

[22] S. K. Kim and M. Baker, Nucl. Phys. B164, 152 (1980).

[23] G. 't Hooft and M. Veltman, Nucl. Phys. B44, 189 (1972); C. G. Bollini and J. J. Giambiagi, Nuovo Cimento 12B, 20 (1972).

[24] L. M. Brown and R. P. Feynman, Phys. Rev. 85, 231 (1952); G. Passarino and M. Veltman, Nucl. Phys. B160, 151 (1979).

[25] B. W. Harris and J. Smith, Phys. Rev. D 51, 4550 (1995).

[26] A. I. Davydychev, Phys. Lett. B 263, 107 (1991).

[27] F. V. Tkachov, Phys. Lett. 100B, 65 (1981); K. G. Chetyrkin and F. V. Tkachov, Nucl. Phys. B192, 159 (1981).

[28] A. I. Davydychev, J. Phys. A 25, 5587 (1992).

[29] A. C. Hearn, REDUCE User's Manual (version 3.5), RAND publication No. CP78, Santa Monica, 1993 (unpublished).

[30] G. Källen, Elementary Particle Physics (Addison-Wesley, Reading, MA, 1964).

[31] H. M. Fried and D. R. Yennie, Phys. Rev. 112, 1391 (1958).

[32] A. A. Abrikosov, Zh. Eksp. Teor. Fiz. 30, 96 (1956) [Sov. Phys. JETP 3, 71 (1956)]; L. D. Soloviev, Dokl. Akad. Nauk SSSR 110, 203 (1956) [Sov. Phys. Dokl. 110, 536 (1957)].

[33] I. Vendramin, Nuovo Cimento A 87, 295 (1985).

[34] W. A. Bardeen, A. J. Buras, D. W. Duke, and T. Muta, Phys. Rev. D 18, 3998 (1978).

[35] E. Braaten and J. P. Leveille, Phys. Rev. D 24, 1369 (1981).

[36] N. I. Ussyukina and A. I. Davydychev, Phys. Lett. B 298, 363 (1993); 305, 136 (1993).

[37] N. I. Ussyukina and A. I. Davydychev, Phys. Lett. B 332, 159 (1994); 348, 503 (1995).

[38] J. S. Ball and T.-W. Chiu, Phys. Rev. D 22, 2542 (1980); L. V. Dung, H. D. Phuoc, and O. V. Tarasov, Sov. J. Nucl. Phys. 50, 1072 (1989); A. Kizılersü, M. Reenders, and M. R. Pennington, Phys. Rev. D 52, 1242 (1995).

[39] E. E. Boos and A. I. Davydychev, Vestn. Mosk. Univ. 28(3), 8 (1987); Teor. Mat. Fiz. 89, 56 (1991) [Theor. Math. Phys. 89, 1052 (1991)].

[40] Higher Transcendental Functions (Bateman manuscript project), edited by A. Erdelyi, W. Magnus, F. Oberhettinger, and F. G. Tricomi (McGraw-Hill, New York, 1953), Vol. 1; A. P. Prudnikov, Yu. A. Brychkov, and O. I. Marichev, Integrals and Series (Gordon and Breach, New York, 1990), 
Vol. 3.

[41] A. I. Davydychev and J. B. Tausk, Phys. Rev. D 53, 7381 (1996).

[42] C. Ford, I. Jack, and D. R. T. Jones, Nucl. Phys. B387, 373 (1992)

[43] R. Scharf, diploma thesis, Würzburg, 1991.

[44] A. I. Davydychev and J. B. Tausk, Nucl. Phys. B397, 123 (1993).

[45] G. 't Hooft and M. Veltman, Nucl. Phys. B153, 365 (1979).
[46] H. J. Lu and C. A. Perez, Report No. SLAC-PUB-5809, 1992 (unpublished).

[47] J. J. van der Bij and M. Veltman, Nucl. Phys. B231, 205 (1984); D. J. Broadhurst, Z. Phys. C 47, 115 (1990).

[48] M. D'Eramo, L. Peliti, and G. Parisi, Lett. Nuovo Cimento 2, 878 (1971).

[49] Z. Bern, L. Dixon, and D. A. Kosower, Nucl. Phys. B412, 751 (1994).

[50] A. I. Davydychev, diploma thesis, MSU, Moscow, 1984. 